\title{
Xiao Yao San Improves Depressive-Like Behavior in Rats through Modulation of $\beta$-Arrestin 2-Mediated Pathways in Hippocampus
}

\author{
Xiaoxia Zhu, ${ }^{1,2}$ Oudong Xia, ${ }^{2,3}$ Weili Han, ${ }^{4}$ Meng Shao, ${ }^{2}$ Linlin Jing, ${ }^{5}$ Qin Fan, \\ Yuanliang Liu, ${ }^{2}$ Jianxin Diao, ${ }^{2}$ Zhiping Lv, ${ }^{1,2}$ and Xuegang Sun ${ }^{1,2}$ \\ ${ }^{1}$ Department of Traditional Chinese Medicine, Nanfang Hospital, Southern Medical University, No. 1838, Guangzhou 510515, China \\ ${ }^{2}$ The Key Laboratory of Molecular Biology, State Administration of Traditional Chinese Medicine, \\ School of Traditional Chinese Medicine, Southern Medical University, Guangzhou, Guangdong 510515, China \\ ${ }^{3}$ Dean's Office, Zhujiang Hospital, Southern Medical University, Guangzhou 510282, China \\ ${ }^{4}$ Hygiene Detection Center, School of Public Health and Tropical Medicine, Southern Medical University, Guangzhou 510515, China \\ ${ }^{5}$ Traditional Chinese Medicine Integrated Hospital, Southern Medical University, Guangzhou, Guangdong 510305, China
}

Correspondence should be addressed to Zhiping Lv; lzp_smu@126.com and Xuegang Sun; sxg_smu@126.com

Received 25 March 2014; Revised 26 May 2014; Accepted 4 June 2014; Published 7 July 2014

Academic Editor: Karl Wah-Keung Tsim

Copyright (C) 2014 Xiaoxia Zhu et al. This is an open access article distributed under the Creative Commons Attribution License, which permits unrestricted use, distribution, and reproduction in any medium, provided the original work is properly cited.

\begin{abstract}
Xiao Yao San (XYS) is a classical Chinese medicine formula that has been widely used to treat mood disorders for hundreds of years. To confirm the effect of XYS and better understand its underlying mechanism, high-performance liquid chromatographymass spectrometry analysis-based quality control of XYS extracts and proteomics-based identification of differential proteins in the hippocampus were adopted in social isolation and chronic unpredictable mild stress- (CUMS-) treated rats. The depressive-like behavior of rats induced by CUMS resembled the manifestation of human depression. The upregulated corticosterone (CORT) and urocortin 2 (UCN2) levels demonstrated the existence of hypothalamic-pituitary-adrenal (HPA) axis hyperactivity. XYS was effective in ameliorating the depressive-like behavior and downregulating UCN2 and CORT. XYS decreased the expression of serine/threonine-protein phosphatase $2 \mathrm{~A}$ subunit $\mathrm{B}$ and increased the expression of $\beta$-arrestin 2 . The expressions of brainderived neurotrophic factor (BDNF), tyrosine receptor kinase B (TrkB), and mammalian target of rapamycin (mTOR) were also elevated by XYS. In conclusion, XYS improves social isolation and CUMS-induced depressive-like behavior and ameliorates HPA hyperactivation through the downregulation of corticotrophin releasing hormone $(\mathrm{CRH})$ receptor 2. The upregulation of $\mathrm{BDNF} / \operatorname{TrkB}$ and the phosphorylation of $\mathrm{mTOR}$ require $\beta$-arrestin 2 as a scaffold to regulate stress signaling.
\end{abstract}

\section{Introduction}

Major depressive disorder (MDD) is a mental disorder characterized by episodes of all-encompassing low mood accompanied by low self-esteem and loss of interest or pleasure in normally enjoyable activities. The prevalence of 12-month and lifetime DSM-IV MDD among adults in the United States is $5.28 \%$ and $13.23 \%$, respectively [1]. MDD ranks second among the diseases with the largest number of years lived with disability and fifth in the disability-adjusted life-year ranking of the top 30 diseases and injuries in 2010 [2]. Longitudinal studies confirm a rising prevalence of MDD, suggesting that we are indeed in the midst of an MDD epidemic [3]. Furthermore, almost half of MDD patients think about suicide or want to die. Therefore, MDD uses many social and health care services and continues to be a serious personal and public health problem.

Serotonin deficiency is the prevailing hypothesis of MDD [4]. Selective serotonin reuptake inhibitors account for about $60 \%$ to $80 \%$ of the market share of antidepressants [5]. However, 5-hydroxytryptamine (5-HT) self-depletion does not cause depression in healthy volunteers and does not worsen symptoms in unmedicated depressed patients [6]. Hypothalamic-pituitary-adrenocortical (HPA) hyperactivity 
is a result of the deficit negative feedback regulation of the axis. HPA is causally linked to the development of depressive symptoms and occurs in $30 \%$ to $50 \%$ of acutely depressed patients [7]. The hippocampus is responsible for the learning and cognition part of the depressive disorder [8]. Neurotrophin deficiency in the hippocampus [9] is involved in the pathogenesis of MDD. However, these various hypotheses are a partial understanding of MDD and are far from being mutually exclusive [10].

Many traditional Chinese herbal formulas have been reported to have antidepressant-like effects [11-14]. Among them, Xiao Yao San (XYS) is the most commonly used formula in treatment of depressive mood disorders for it has been widely prescribed for about nine hundred years from the Song dynasty. MDD patients usually manifest liver stagnation and spleen deficiency according to theory of traditional Chinese medicine. XYS can soothe the liver, invigorate the spleen, and nourish the blood so it has been widely used to treat MDD. Moreover, XYS upregulates the 5-HT content [15], downregulates corticotropin-releasing hormone (CRH) expression in the hypothalamus [16], and increases the expression of brain-derived neurotrophic factor (BDNF) in the hippocampus [17]. However, the mechanism of XYS and the pathophysiology of MDD remain ambiguous. A proteomics-based approach was used to identify the differentially expressed hippocampal protein profiles of XYS in a depressive model induced by social isolation and chronic unpredictable mild stress (CUMS). This study aimed to establish a more integrative framework for MDD by unifying HPA hyperactivity and hippocampal neuron deficiency and to provide a deeper understanding of the mechanism of XYS.

\section{Materials and Methods}

2.1. Preparation of Drugs. XYS is composed of Bupleuri Radix (root of Bupleurum Chinese DC), Angelicae sinensis Radix (root of Angelica sinensis (Oliv.) Diels), Paeoniae Radix Alba (root of Paeonia lactiflora Pall.), Atractylodis Macrocephalae Rhizoma (root and rhizome of Atractylodes macrocephala Koidz), Poria (fungus nucleus of Poria $\operatorname{cocos}$ (Schw.) Wolf), Zingiberis Rhizoma Recens (root and rhizome of Zingiber officinale Rosc.), Menthae Haplocalycis Herba (overground parts of Mentha haplocalyx Briq.), and Glycyrrhizae Radix et Rhizoma (root and rhizome of Glycyrrhiza uralensis Fish.). The raw herbs for XYS were purchased from the Affiliated Nan Fang Hospital of Southern Medical University. The herb materials were authenticated by Professor Liu Q, an expert on pharmacognostical identification in School of Traditional Chinese Medicine, Southern Medical University. The voucher specimens were deposited in the storage cabinet of Chinese traditional medicine of School of Traditional Chinese Medicine, Southern Medical University. A total of $185 \mathrm{~g}$ of herbs was mixed at a ratio of $6: 6: 6: 6: 6: 2: 2: 3$ (dry weight). Aqueous extracts of XYS were extracted at $80^{\circ} \mathrm{C}$ by stirring for $1 \mathrm{~h}$ using 10 volumes of distilled water $(\mathrm{v} / \mathrm{m})$. The extracts were centrifuged at $1,500 \times \mathrm{g}$ at room temperature. The supernatant was collected and subjected to condensation under reduced pressure at $70^{\circ} \mathrm{C}$ to obtain the semisolid
XYS solution [18]. Quality of XYS was confirmed by highperformance liquid chromatography-mass spectrometry analysis (see Figure S1 in Supplementary Materials available online at http://dx.doi.org/10.1155/2014/902516). XYS was suspended again in distilled water at a final concentration of $1.9 \mathrm{~g} / \mathrm{mL}$. Fluoxetine hydrochloride (Lilly Suzhou Pharmaceutical Co., LTD, number J20080016) was purchased from the Affiliated Nan Fang Hospital of Southern Medical University and dissolved in distilled water to a final concentration of $0.2 \mathrm{mg} / \mathrm{mL}$. The solution was stored in aliquots at $-20^{\circ} \mathrm{C}$.

2.2. Animals and Experimental Procedures. All procedures involving laboratory animals were in accordance with the guidelines of the Instituted Animal Care and Use Committee of Southern Medical University. All protocols were submitted and validated by the Animal Care Ethics Committee of Southern Medical University (number 2012-065). A total of 40 male Sprague-Dawley rats, weighing $200 \pm 20$ g, were purchased from the Center of Experimental Animals, Southern Medical University. The animals were maintained under controlled conditions $\left(22^{\circ} \mathrm{C}, 12 \mathrm{~h} / 12 \mathrm{~h}\right.$ dark/light cycle $)$ in a conventional animal colony for one week to adapt to the new environment.

Rats were assigned randomly into four groups: control, model, XYS, and fluoxetine. Five animals per cage in the control group were housed and allowed free access to food and water. Animals in the other three groups underwent social isolation and CUMS procedures (Table S1). Briefly, each animal was socially isolated by placing each animal in a separate cage and underwent CUMS protocol. CUMS procedure involves a variety of mild stressors: (1) food deprivation for $24 \mathrm{~h}$, (2) water deprivation for $24 \mathrm{~h}$, (3) exposure to an empty bottle for $1 \mathrm{~h}$, (4) cage tilt $\left(45^{\circ}\right)$ for $7 \mathrm{~h}$, (5) overnight illumination, (6) soiled cage $(200 \mathrm{~mL}$ of water in $100 \mathrm{~g}$ of sawdust bedding) for $24 \mathrm{~h},(7)$ forced swimming at $8^{\circ} \mathrm{C}$ for $30 \mathrm{~min}$, (8) physical restraint for $3 \mathrm{~h}$, and (9) exposure to a foreign object (e.g., a piece of plastic) for $24 \mathrm{~h}$ [19]. About $19 \mathrm{~g} / \mathrm{kg} / \mathrm{d}$ XYS, $2 \mathrm{mg} / \mathrm{kg} / \mathrm{d}$ fluoxetine, and an equivalent volume of distilled water (for model and control groups) were administrated by gavage using a tube twice a day.

2.3. Behavior Tests. The open-field apparatus was a four-sided $80 \times 80 \times 40 \mathrm{~cm}$ wooden enclosure, with the side walls painted black and the floor painted khaki divided into 25 equal squares by black lines. The open-field tests were performed in a dimly lit $(25 \mathrm{~W})$ and quiet (less than $60 \mathrm{~dB}$ ) room. Each rat was gently placed at the center of the square and observed for consecutive 5 min periods. All animals were tested once between 9 am and $1 \mathrm{pm}$. The apparatus was wiped with $70 \%$ ethanol and dried between rats. For scoring, a video-tracking system (EthoVision, Noldus, Wageningen, Holland) was used to record the grids travelled and the number of rears [20].

Sucrose preference test was performed after rats were provided with a free choice between two bottles (one with $1 \%$ sucrose solution and another with tap water) for $24 \mathrm{~h}$. The position of the bottles was switched after $12 \mathrm{~h}$ to prevent the possible effects of side preference in drinking behavior. No previous food or water deprivation was applied before the 
test. The consumption of water and sucrose solution was estimated simultaneously in control and experimental groups by weighing the bottles. The sucrose intake was calculated as an amount of consumed sucrose in grams. The preference for sucrose was calculated as the percentage of the consumed sucrose solution of the total amount of liquid drunk [21].

Food consumption evaluations were made after fasting for $24 \mathrm{~h}$. This further evaluation was necessary because food deprivation was used in our behavioral tasks as a mild stressor [22].

Body weight was measured and recorded on the last day of the week.

The rats were sacrificed after the behavioral tests. The whole brains of three rats in each group were removed after accepting heart perfusion. The brains were fixed in $4 \%$ paraformaldehyde solution for further histopathological assessment and immunohistochemistry. The left hippoacmpi of the remaining rats were flash-frozen in liquid nitrogen and stored at $-80^{\circ} \mathrm{C}$ for protein analysis. The right hippocampi were kept in 10 volumes of RNAlater Solution (Ambion, Life technologies, Carlsbad, CA, USA) for polymerase chain reaction (PCR) analysis.

2.4. Hormone Measurement. Blood was collected from the abdominal aorta by a puncturing needle. Cerebrospinal fluid (CSF) was extracted from the foramen magnum by a $1 \mathrm{~mL}$ syringe. Blood samples were placed at room temperature for $30 \mathrm{~min}$ before centrifugation for $10 \mathrm{~min}$ at $3,000 \times \mathrm{g}$.

Serum adrenocorticotropic hormone (ACTH) and corticosterone (CORT) were analyzed using an IMMULITE 2000 immunoassay system with ACTH and CORT immunoassay kits.

The serum and the CSF levels of CRH and urocortin-2 (UCN2) were detected by a microtiter plate reader $\left(\right.$ Victor $3_{\mathrm{V}}$, Perkin Elmer, Waltham, MA, USA) with rat CRH enzymelinked immunosorbent assay (ELISA) kit (Baoman Biotech, Shanghai) and rat UCN2 ELISA kit (Chang Yi Chemical, Chemical, Shanghai).

2.5. Histopathological Assessment and Nissl's Staining. In the histopathological examination, paraformaldehyde solution was used to fix the paraffin-embedded brain tissues that were cut into serial sections $(3 \mu \mathrm{m})$. The slices were stained with hematoxylin and eosin and Nissl's solution in a routine procedure.

2.6. Two-Dimensional Electrophoresis (2D) and Protein Identification. The $2 \mathrm{D}$ electrophoresis was performed as previously reported and repeated for three times [18]. Briefly, hippocampal samples containing $300 \mu \mathrm{g}$ of protein were loaded per tube in an isoelectric focusing system (IPGphor II, GE). The samples were isoelectrofocused and separated with sodium dodecyl sulfate-polyacrylamide gel (SDS-PAGE). The gels were silver-stained. The protein spots that either increased or decreased for more than twofold were selected for matrix-assisted laser desorption/ionization time of flight mass spectrometry (MALDI-TOF MS) identification. The mascot software package and the database of SwissProt were used to match the mass of peptides [23].

2.7. Western Blot Analysis. Western blot analysis was performed as previously described [24]. Briefly, the protein lysates were loaded onto $10 \%$ SDS-PAGE for separation, electrotransferred onto PVDF membranes, and blocked in 5\% nonfat milk in Tris-buffered saline Tween. The membranes were incubated overnight with primary antibodies, such as anti- $\beta$-arrestin 2 (Cell Signaling Technology, CST, clone C16D9, 1:750 dilution), anti-extracellular signal-regulated kinase (ERK, CST, Clone 137F5, 1:800 dilution), antiphospho-ERK (Thr202/Tyr204, CST, 1:800 dilution), antityrosine receptor kinase B (TrkB, Bioss, clone bs-0175R, 1:400 dilution), anti-BDNF (epitomics, 1:4000 dilution), anti-serine/threonine-protein phosphatase $2 \mathrm{~A}$ subunit $\mathrm{B}$ (PP2A b, Abcam, 1:800 dilution), anti-PP2A c (Abcam, 1:750 dilution), anti-CRH receptor 1 (CRHR1, Bioss, 1:400 dilution), anti-CRH receptor 2 (CRHR2, Abcam, $1: 800$ dilution), anti-mammalian target of rapamycin (mTOR, CST, 1:800 dilution), anti-phospho-mTOR (phospho-S2448, Abcam, $1: 800$ dilution), and anti- $\beta$-actin (Clone TA-09, ZSGB-BIO, $1: 1000)$ at $4^{\circ} \mathrm{C}$. This procedure was followed by incubation with horseradish peroxidase- (HRP-) conjugated secondary antibody. The results were visualized with enhanced chemiluminescence (GE Healthcare Bio-science, Uppsata, Sweden). Images were captured and documented with a CCD system (Imagestation 2000MM, Kodak, Rochester, Rochester, NY, USA). The quantitative analysis of these images was performed using Molecular Imaging Software Version 4.0 (provided by Kodak 2000MM System). The optical density was normalized against that of the $\beta$-actin.

2.8. Immunohistochemistry. The paraffin-embedded hippocampal sections were deparaffinized, rehydrated, and pretreated with hydrogen peroxidase in phosphate buffer solution. Heat-induced antigen retrieval was conducted. After blocking with the appropriate antisera, sections were incubated with anti- $\beta$-arrestin 2 (CST, 1:50 dilution), antiBDNF (epitomics, 1:100 dilution), anti-TrkB (Bioss, 1:100 dilution), anti-phospho-ERK (Thr202/Tyr204, CST, 1:50 dilution), anti-CRHR1 (Bioss, 1:100 dilution), and antiCRHR2 (Abcam, 1:50 dilution). After incubation with HRPconjugated secondary antibody and tyramide amplification followed by streptavidin-HRP, positive signals were visualized by a diaminobenzidine kit and counter-stained with hematoxylin.

2.9. Quantitative RT-PCR Detection. RNA isolation and quantitative real-time PCR (qRT-PCR) were performed as described previously [25]. Briefly, total RNA from each indicated group was extracted using Trizol reagent. Complementary DNA was synthesized, and qRT-PCR was performed on a Stratagene Mx3005P QPCR System (La Jolla, CA, USA). The sequences of primers are listed in Table S2. PCR results were analyzed using Opticon Monitor Analysis 2.0 software (Bio-Rad Laboratories, Hercules, CA, USA). Relative mRNA expression was quantified by subtracting the glyceraldehyde 
3-phosphate dehydrogenase threshold cycle $\left(C_{t}\right)$ value from the $C_{t}$ value of the genes of interest. It is expressed as $2^{-\Delta \Delta C_{t}}$.

2.10. Statistical Analysis. All data were expressed as mean \pm SD and analyzed using an SPSS statistical package (version 13.0, Armonk, NY, USA). Mean values were compared through one-way ANOVA. A multicomparison was also conducted. Data were tested through homogeneity test for variance. The mean values of the homogenous variances were compared using ANOVA. The differences between the two groups were analyzed based on the test of least significance difference. The mean values of the nonhomogeneous variances were compared using Welch's test. The differences between the two groups were analyzed using the GamesHowell test. $P<0.05$ was considered statistically significant.

\section{Results}

3.1. Effect of XYS on Behavioral Changes in Socially Isolated and CUMS-Induced Depressive Rats. All rats were active, were brisk, and were in good mood fitness with white and lustrous furs before the experiment. In the beginning, the rats were agitated when restrained and frequently groomed after being unclenched. Repeated struggling was observed when the rats were forced to swim. After $10 \mathrm{~d}$, the rats in the model group became numb and showed unnatural passivity manifested as being lazy and sluggish and dodging the nooks. At the end of the $21 \mathrm{~d}$ of CUMS, the rats showed various depressive behaviors, such as poor appetite, droopy whiskers, lowpitched voice, lusterless fur, loose stool, and slow responsiveness. XYS treatment ameliorated the depressive behavior by improving the appetite and responsiveness of the rats.

Social isolation and CUMS treatment decreased the body weight of rats at $14 \mathrm{~d}$ and $21 \mathrm{~d}(P<0.01)$. Moreover, XYS and fluoxetine significantly improved the body weight loss at $14 \mathrm{~d}$ and $21 \mathrm{~d}(P<0.01)$ (Figure $1(\mathrm{a}))$.

At $21 \mathrm{~d}$, the number of grids crossed with all paws, and the number of rearing responses significantly decreased in the model group unlike in the control $(P<0.01)$. XYS had no significant effects on rats without social isolation and CUMS treatment (Figure S2). XYS and fluoxetine significantly increased the number of crossed grids $(P<0.05)$, and XYS increased the rearing responses $(P<0.01)$ (Figures $1(\mathrm{~b})-$ $1(\mathrm{~d})$ ).

Social isolation and CUMS significantly decreased the percentage of sucrose consumption in the model group unlike the control group $(P<0.01)$. Long-term treatment with XYS and fluoxetine significantly increased the percentage of sucrose consumption in socially isolated and CUMSexposed rats $(P<0.01)$ unlike the model group (Figure 1(e)). Moreover, food consumption significantly decreased unlike the control group $(P<0.01)$. XYS significantly increased food consumption $(P<0.05)$, whereas fluoxetine failed to increase food consumption unlike the model group (Figure 1(f)).

3.2. Effect of XYS on ACTH, CORT, CRH, and UCN2 in Socially Isolated and CUMS-Induced Depressive Rats. The serum CORT significantly increased in the model group unlike the control group $(P<0.05)$; serum and CSF UCN2 also increased $(P<0.01)$. XYS and fluoxetine significantly decreased the CORT level $(P<0.05)$ as well as the serum and CSF UCN2 levels $(P<0.01)$. No significant difference was observed in the serum and CSF levels of $\mathrm{CRH}$ and serum ACTH based on the ANOVA (Figure 2).

3.3. Effect of XYS on Morphology and Neuron Number in the Hippocampus. HE staining showed that neuronal cells in the hippocampus were neatly arranged in the control group (Figure 3(a)). Neuronal cell bodies and basophilic granules decreased in the model group. The neuronal cell numbers improved, and Nissl's body was increased by XYS (Figure 3(b)).

3.4. Effects of XYS on the Expressions of CRHR1 and CRHR2. The expression of CRHR2 was upregulated in the model group unlike the control group. The expression was significantly downregulated by XYS and fluoxetine treatment. No significant difference was observed in the expression of CRHR1 among the four groups (Figure 4).

3.5. Proteomics Analysis of Differentially Expressed Phosphorylated Proteins. Based on the reproducible 2D gel electrophoresis, two downregulated and eight upregulated spots were observed in the model group unlike the control group (Figure 5(a)). The two upregulated spots by XYS were identified as prohibitin and proteasome subunit beta type- 6 . Among the eight spots downregulated by XYS, five spots were identified as tubulin alpha-1A chain, DCN1-like protein 3, serine/threonine-protein phosphatase $2 \mathrm{~A} 55 \mathrm{kDa}$ regulatory subunit B alpha isoform (PP2Ab), tubulin beta-2C chain, sodium channel, and clathrin linker 1 (Table 1). Figure 5(b) illustrates the peptide mass fingerprint obtained from the seven spots. Figure 5(c) shows the mascot research results.

3.6. Verification of XYS on the Expression of PP2Ab and on the Phosphorylation of Akt and mTOR. The expression of PP2Ab was increased by social isolation and CUMS treatment; the expression of PP2Ac was not affected. Quantitative RT-PCR also showed that PP2Ab, not PP2Ac, was downregulated by XYS. The phosphorylation of $\mathrm{mTOR}$ and protein kinase $\mathrm{B}$ (Akt) was significantly upregulated by XYS (Figure 6).

3.7. Effect of XYS on the Expressions of $\beta$-Arrestin 2 and BDNF and on the Phosphorylation of ERK. The decreased expression of $\beta$-arrestin 2, BDNF, TrkB and the phosphorylation of ERK was observed in the model group unlike the control group. XYS increased the expression of $\beta$-arrestin $2, \mathrm{BDNF}$, and TrkB and the phosphorylation of ERK. The expression patterns of BDNF, TrkB, and $\beta$-arrestin 2 and the phosphorylation of ERK were further confirmed by immunohistochemistry (Figure 7). 


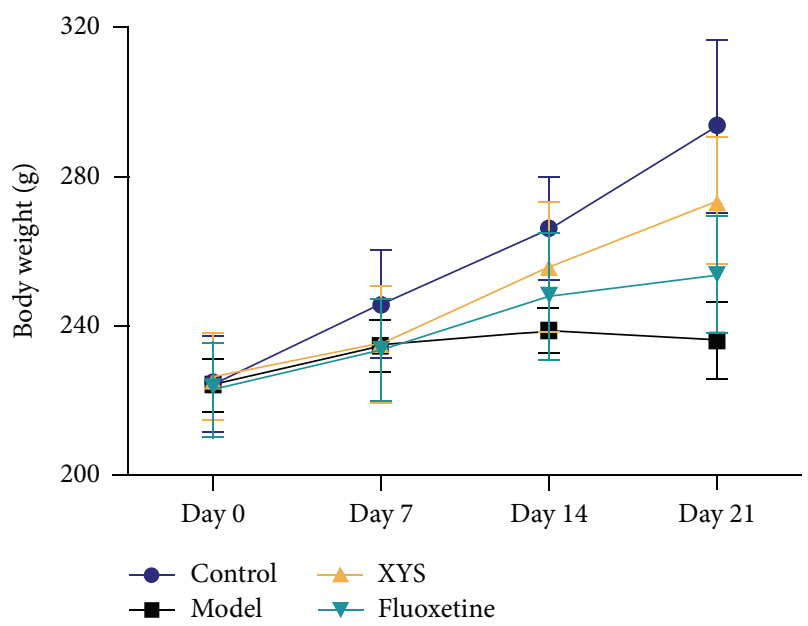

(a)

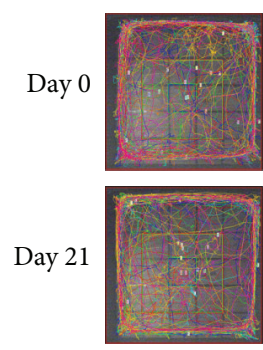

Control
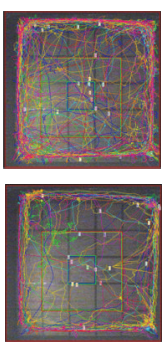

Model

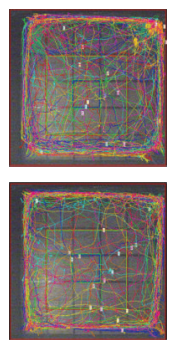

XYS

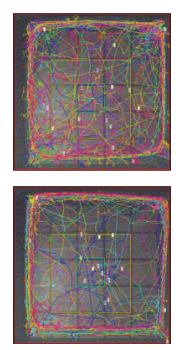

Fluoxetine (b)

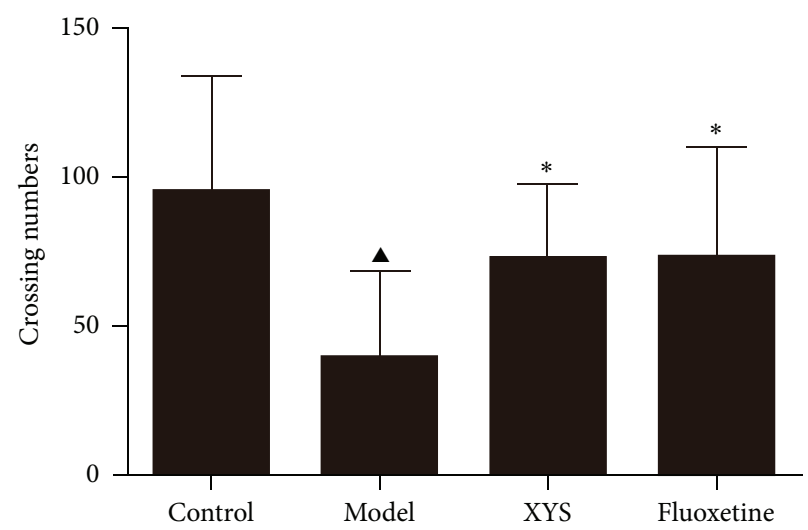

(c)

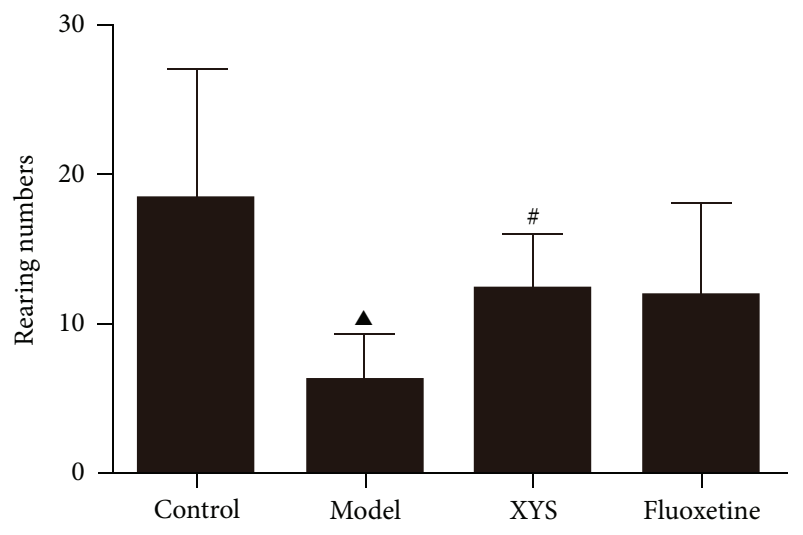

(d)

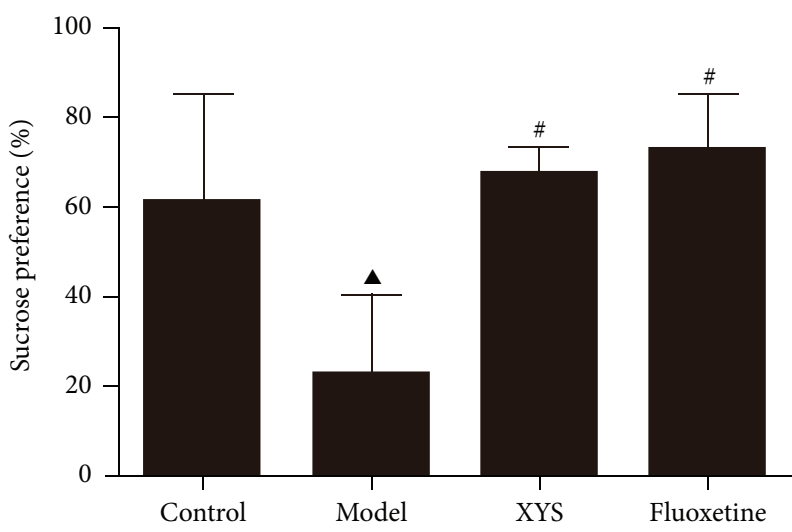

(e)

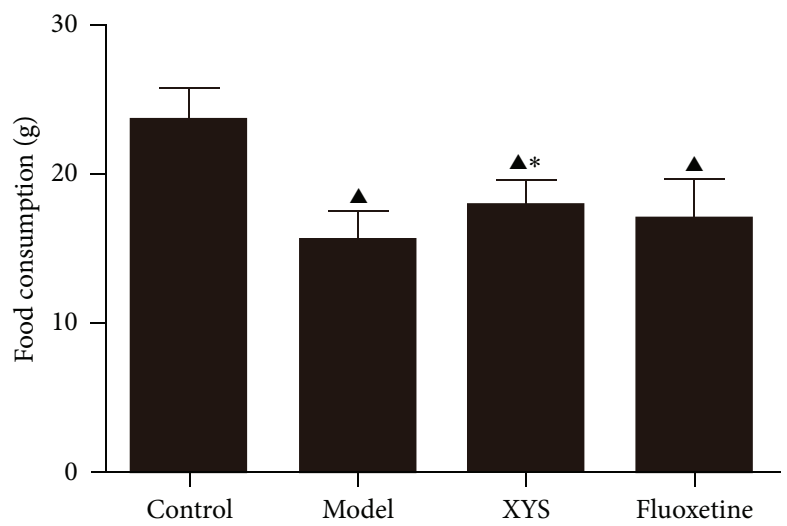

(f)

FIGURE 1: Effects of XYS on body weight and behavior of rats treated with social isolation and CUMS. Body weight was measured once a week (a). A battery of behavioral tests was initiated $21 \mathrm{~d}$ after modeling, and the following parameters were measured: crossing trajectories (b), crossing numbers (c), rearing numbers (d), sucrose preference (e), and food consumption (f). Data are expressed as mean \pm SD, $n=10$ per group. ${ }^{\triangle} P<0.05,{ }^{\wedge} P<0.01$ versus control, ${ }^{*} P<0.05,{ }^{\#} P<0.01$ versus model. 


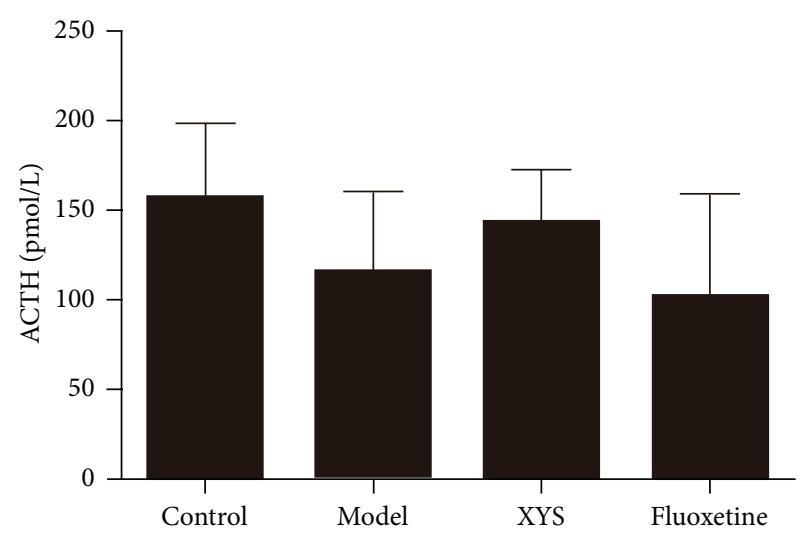

(a)

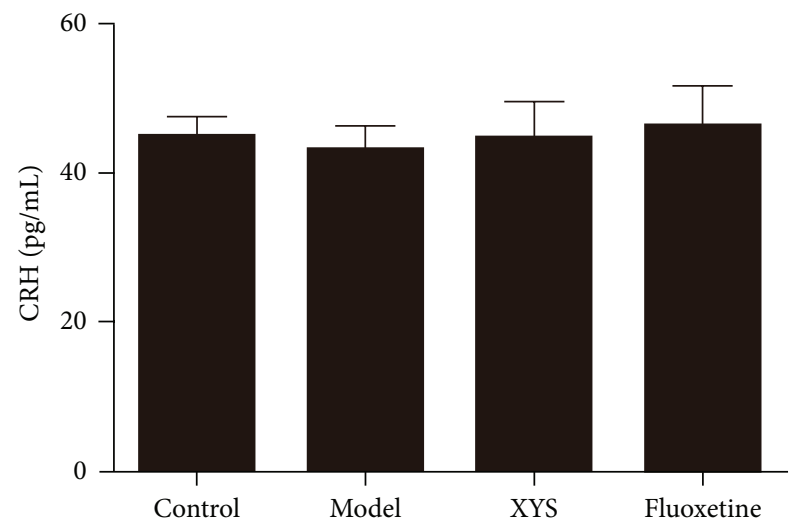

(c)

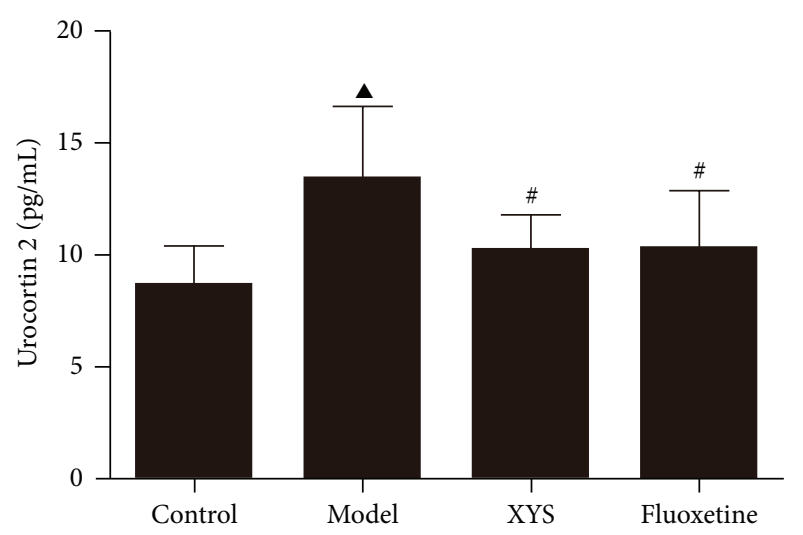

(e)

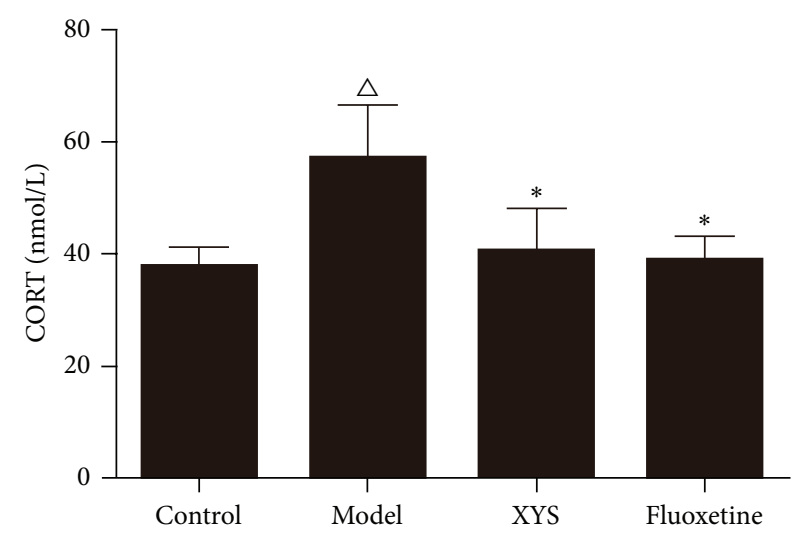

(b)

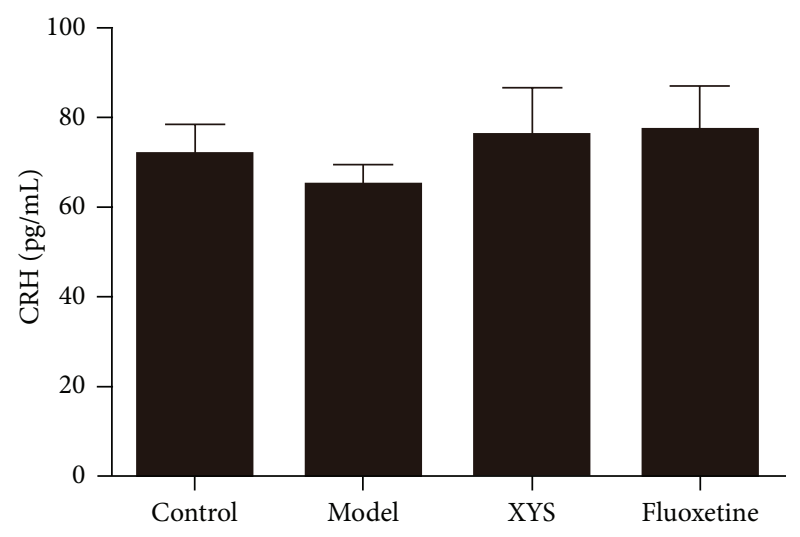

(d)

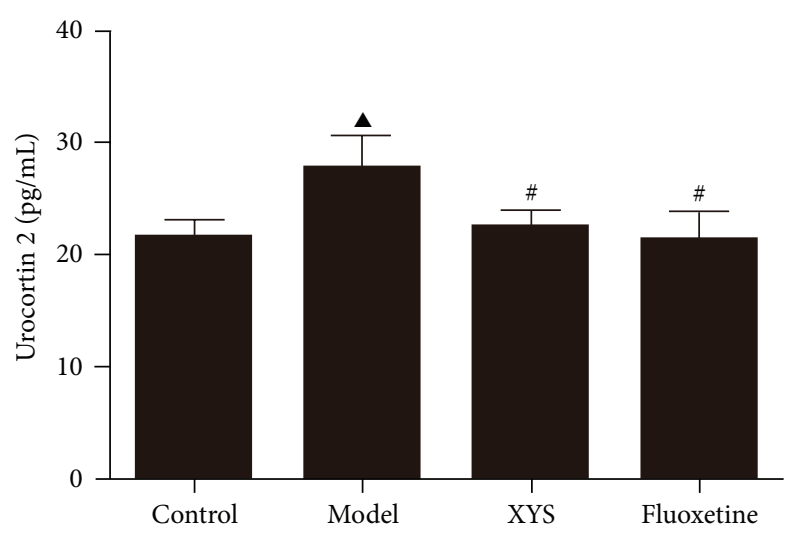

(f)

FIGURE 2: Effects of XYS on serum and cerebrospinal fluid hormone levels in depressive rats. Effect of XYS on serum ACTH (a), serum CORT (b), serum CRH (c), CSF CRH (d), serum urocortin 2 (e), and CSF urocortin (f). Data are expressed as mean \pm SD, $n=6$ per group. ACTH: adrenocorticotropic hormone; CORT: corticosterone; CRH: corticotropin-releasing hormone; CSF: cerebrospinal fluid. ${ }^{\triangle} P<0.05$, ${ }^{\Delta} P<0.01$ versus control, ${ }^{*} P<0.05,{ }^{\#} P<0.01$ versus model.

\section{Discussion}

An animal model of CUMS-induced depression was originally established by Katz [26] and modified by Willner [27] to simulate the pathogenesis of depression in humans. The CUMS paradigm causes anhedonia, which is the loss of interest in normally pleasurable and rewarding activities.
The use of the model is a well-validated method to cause depression, and the model has face and predictive validity [27]. Social isolation aggression can potentiate anxiety and depressive-like behavior in isolated mice subjected to unpredictable chronic mild stress [28]. Therefore, social isolation combined with CUMS is adopted to induce the depressive behavior in rats. Poor appetite, droopy whiskers, 

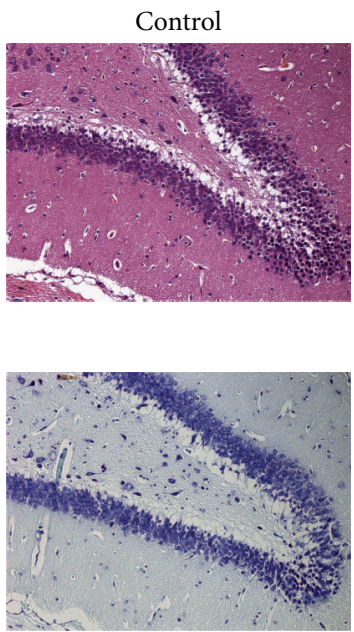
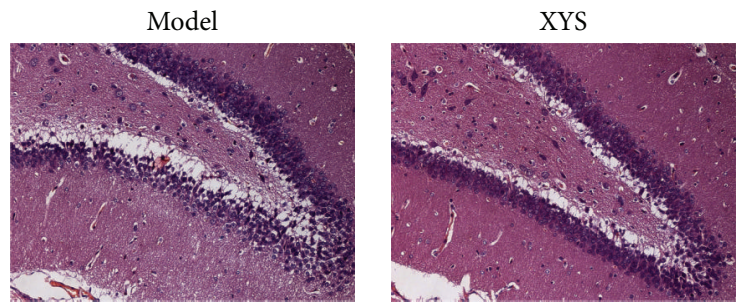

(a)
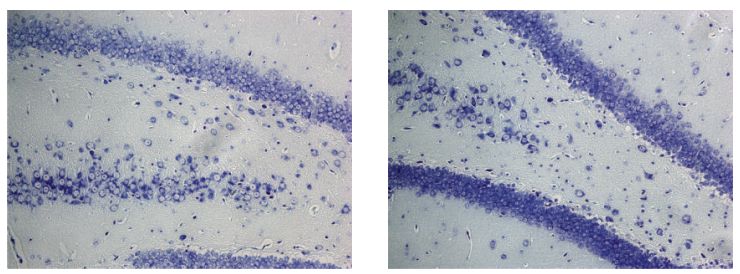
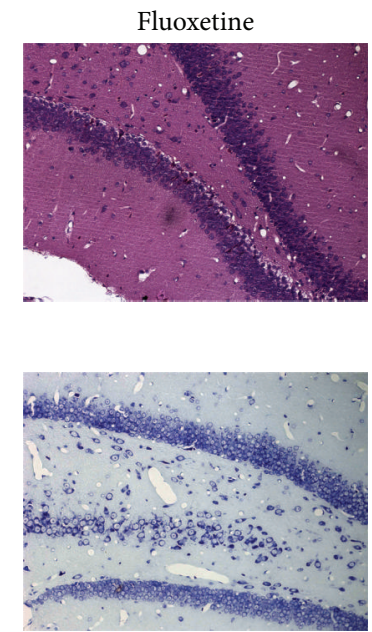

(b)

FIGURE 3: Effect of XYS on the histologic structure of dentate gyrus (DG). HE staining (a) and Nissl's staining of the DG hippocampus (b).
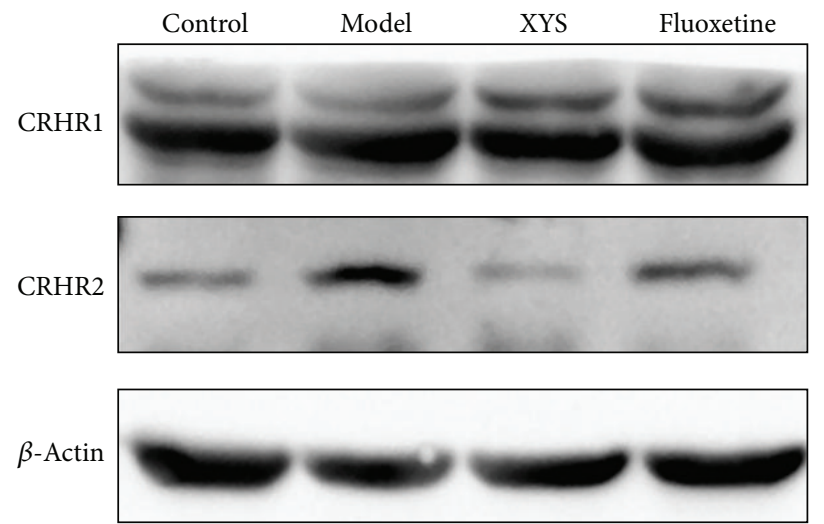

(a)
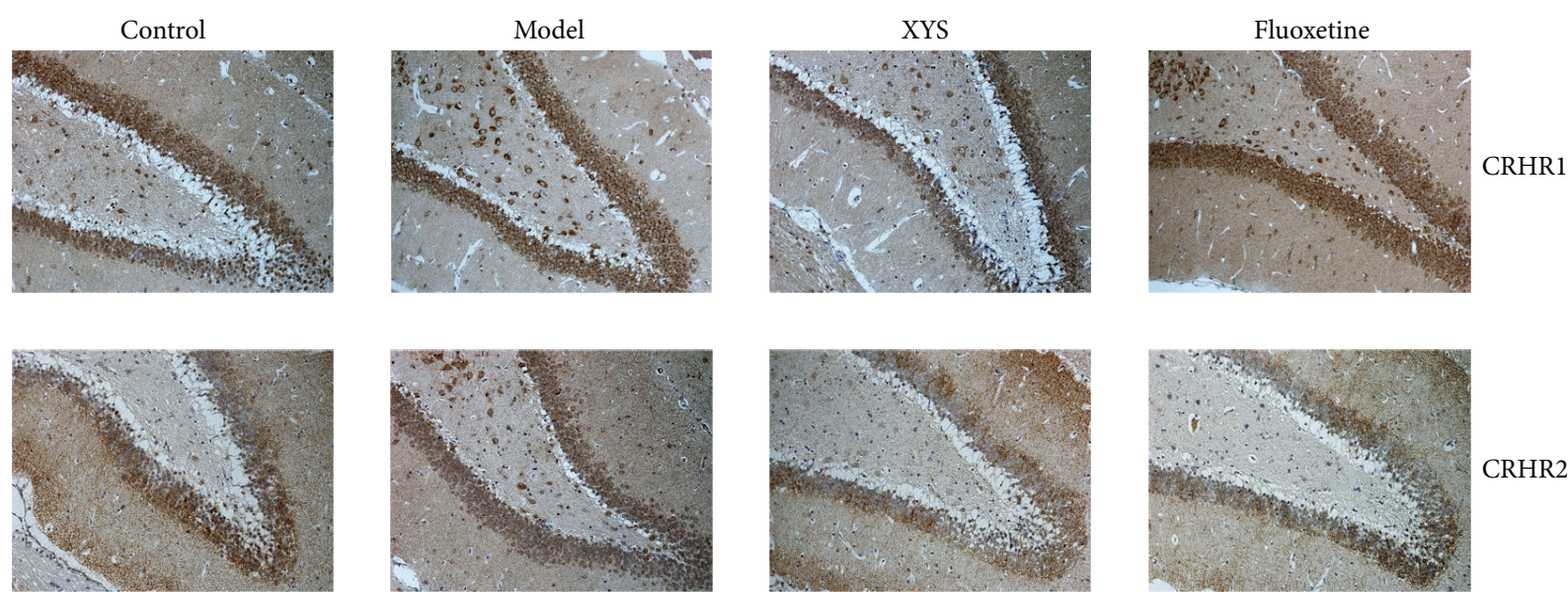

CRHR2

(b)

FIGURE 4: Representative Western blot analysis (a) and immunohistochemical staining (b) of CRHR1 and CRHR2 in the hippocampus. CRHR: corticotropin-releasing hormone receptor. Refer to Table 2 for the semiquantitative analysis of the above images. 

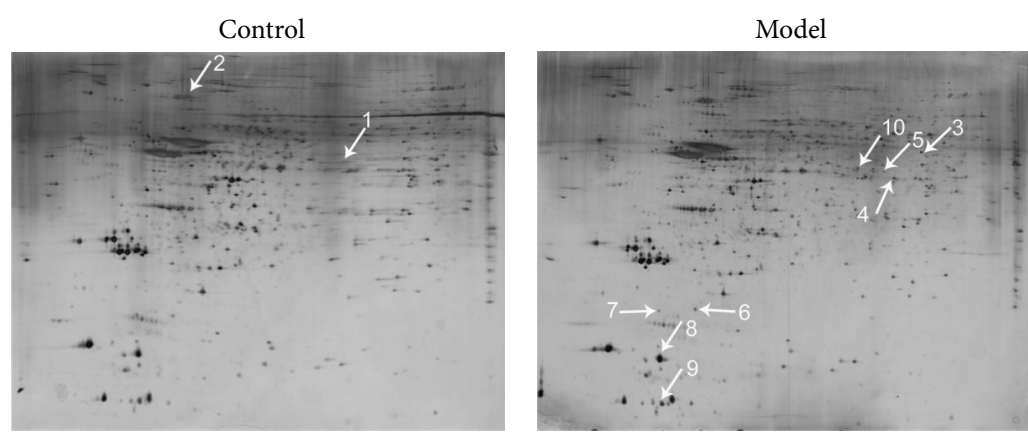

(a)

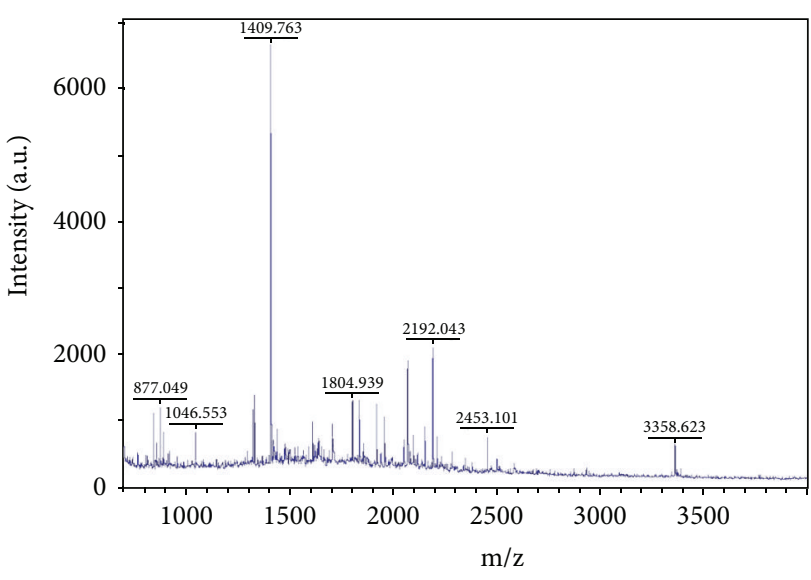

(b)

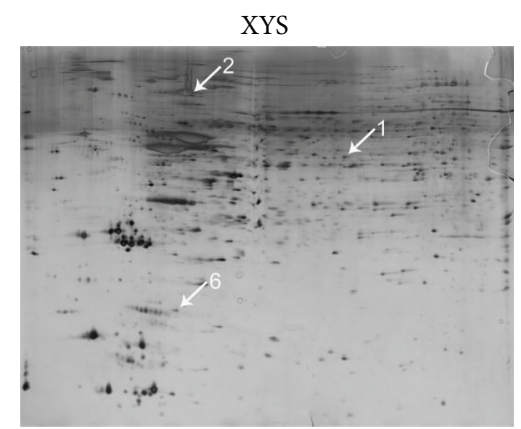

Probability based Mowse score

Protein score is $-10 * \log (P)$, where $P$ is the probability that the observed match is a random event Protein scores greater than 51 are significant $(P<0.05)$

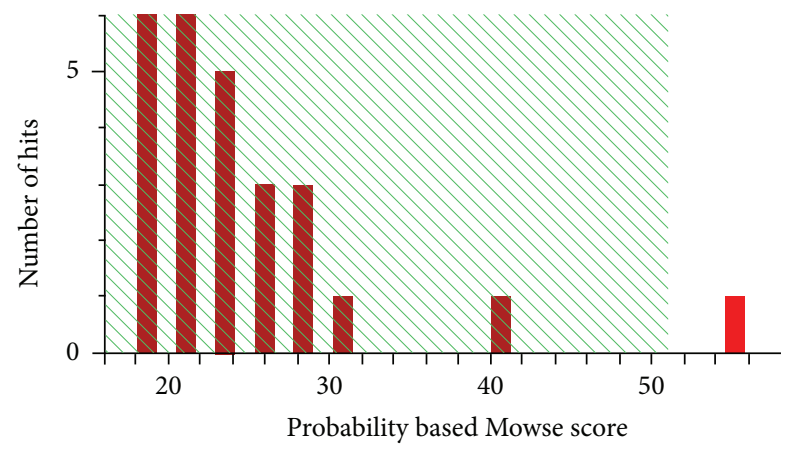

(c)

FIGURE 5: 2D gel images of protein expression and MALDI-TOF MS identification of samples from the hippocampus. 2D gel image of protein expression (a). Mascot search results of spot 7 (b). MALDI-TOF MS was obtained from spot 7 after trypsin digestion (c). 2DE, 2D electrophoresis, MALDI-TOF MS, and matrix-assisted laser desorption/ionization time of flight mass spectrometry.

slow responsiveness of rats, elevated CORT and UCN 2, open field changes, and anhedonia deficiency suggest that socially isolated and CUMS-treated rats exhibit the manifestation of human depression. The tonifying spleen function of XYS may increase the food consumption and body weight of rats [29]. XYS increases locomotor activity, ameliorates anhedonia, and improves neuroendocrine function and appetite, suggesting that XYS is effective in the prevention and treatment of social isolation and CUMS-induced depression.

In the past decades, evidence has shown the association of MDD with small hippocampal volumes [30, 31]. Structural changes in the volume of the hippocampus [32] and neuronal cell death and abnormal synaptic plasticity in the hippocampus [33] have important functions in the pathophysiology of MDD. Nissl's staining confirmed the injuries of the hippocampal neuronal cell bodies in the model group. The HPA axis is governed by the secretion of CRH from the hypothalamus, and it then stimulates the secretion of CORT from the adrenal cortex [34]. A significant elevation of CRHR2, a high-affinity and membrane-bound receptor for UCN2 [35], was observed in the model group but not CRHR1. CRHR2 signals strongly after binding with urocortin, whereas weak CRHR2 activation occurs only if CRH is released [34]. UCN2 can increase the CORT level in rats treated with the fear-conditioning test [36]. Thus, increased serum UCN 2 and serum CORT were observed in the model group. Selective CRHR2 activation also suppresses the exploration and certain locomotor behavior in rodents [37]. XYS may decrease UCN2 and CRHR2 to ameliorate the depressive-like behavior in rats.

Further proteomic research indicates that PP2A is upregulated in the model group and downregulated by XYS. PP2A is a multimeric protein complex composed of a structural $\mathrm{A}$ subunit, a catalytic $\mathrm{C}$ subunit, and a variety of targeting $\mathrm{B}$ subunits [38]. Previous study shows that social isolation negatively regulates Akt [39] and that the activation of Akt may enhance synaptic plasticity through mTOR $[40,41]$. Thus, the phosphorylation of Akt and the expression of mTOR were evaluated. The upregulated phosphorylation of Akt and the expression of mTOR suggest that XYS may improve depressive-like behavior through the PP2A-mediated Akt pathway. Remarkably, $\beta$-arrestin 2 has an essential function in mediating the interaction of Akt with a targeting $B$ subunit and a catalytic $\mathrm{C}$ subunit of PP2A in response to DA [42]. The formation of a protein complex, which is composed of Akt, $\beta$-arrestin 2, and multimeric protein phosphatase PP2A, is essential for the dephosphorylation and the inactivation of Akt. In vitro and in vivo tests show the antidepressant lithium 


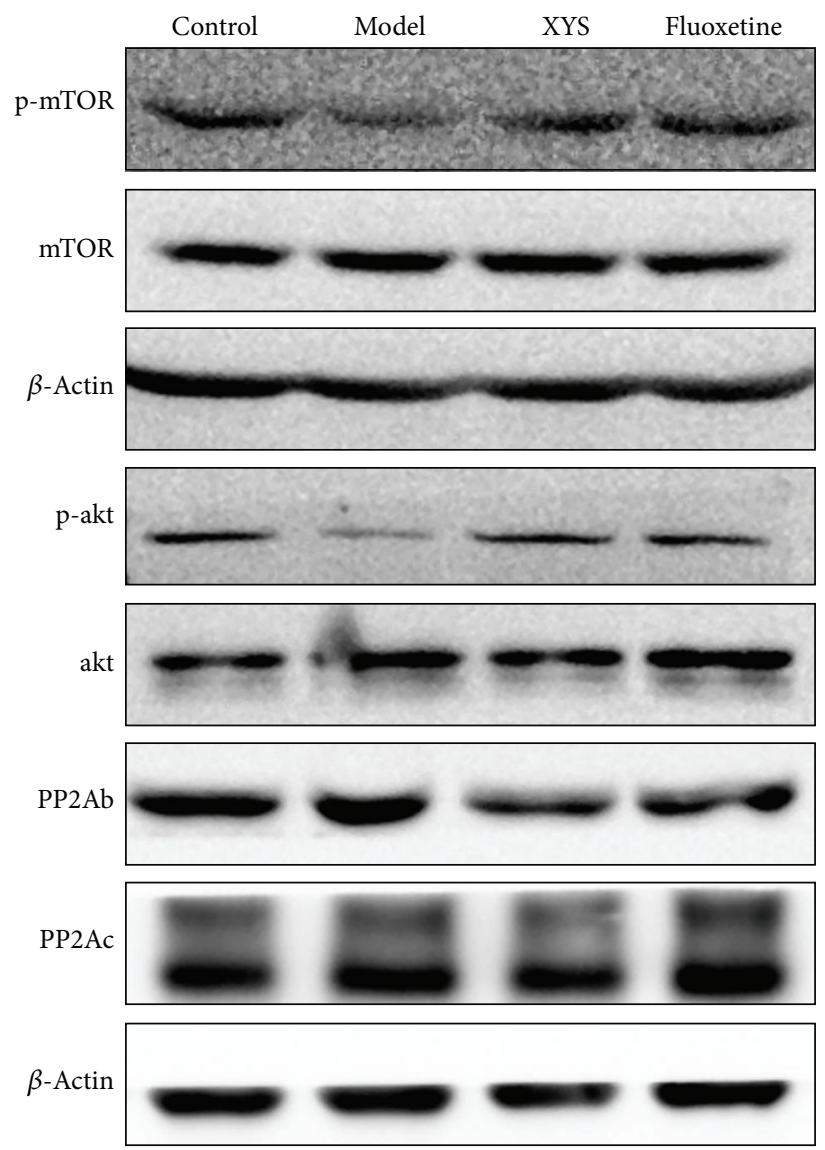

(a)

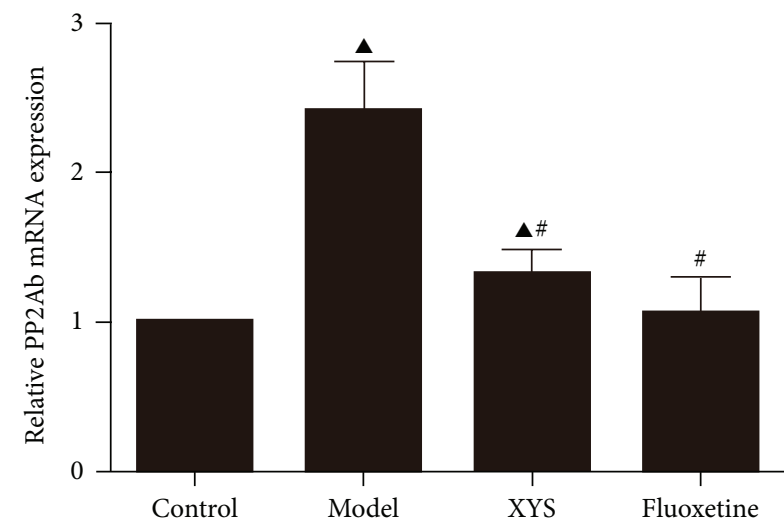

(b)

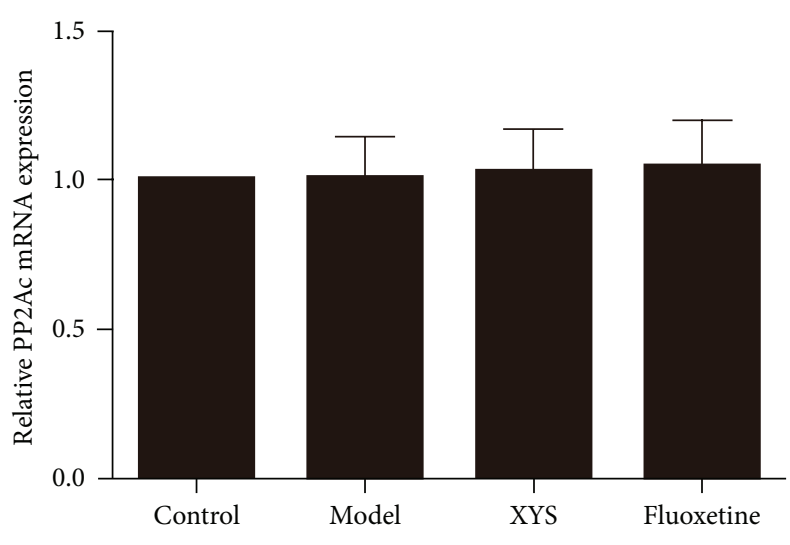

(c)

Figure 6: Representative images of the expressions of phospho-mTOR, phospho-Akt, PP2Ab, and PP2Ac in the hippocampus (a), PP2Ab (b), and PP2Ac (c). mRNA expression levels of the hippocampus by qPCR. p-mTOR: phospho-mammalian target of rapamycin; PP2A: serine/threonine-protein phosphatase $2 \mathrm{~A}$. Refer to Table 2 for the semiquantitative analysis of the above images.

disruption of the Akt: $\beta$-arrestin 2:PP2A signaling complex $[43,44]$. Thus, the function of $\beta$-arrestin 2 in social isolation and CUMS-induced depressive-like behavior was assessed.

$\beta$-arrestin 2 was downregulated in the model group but was improved by XYS and fluoxetine treatment. $\beta$-arrestin 2 can uncouple CRHR, a G protein-coupled receptor (GPCR), from $G$ proteins and promote its internalization, resulting in desensitization and downregulation [45]. Thus, the decreased expression of CRHR2 might be a result of increased desensitization regulated by $\beta$-arrestin 2 [46]. $\beta$-arrestin 2 also functions as a scaffold protein that interacts with several cytoplasmic proteins and links GPCRs to intracellular signaling pathways, such as mitogen-activated protein kinase (MAPK) and Akt [47-49]. $\beta$-arrestin 2 is a positive mediator of dopaminergic synaptic transmission [42]. The phosphorylation of Akt 308 is upregulated in wild-type mice and downregulated in $\beta$-arrestin 2 knockout mice upon lithium chloride treatment [43]. Therefore, XYS may increase the expression of $\beta$-arrestin 2 as a scaffold to enhance the phosphorylation of Akt.
Isolated rats showed a significant decrease in BDNF protein concentrations in the hippocampus [50]. Previous studies also show that phosphorylation of ERK1/2 is downregulated in the hippocampus and prefrontal cortex in rats with depressive-like behavior induced by chronic forced swim stress [51]. Thus, isolation and CUMS might jointly contribute to the downregulation of BDNF in the hippocampus. Fluoxetine alleviates the depressive-like behavior by increasing the phosphorylation of ERK1/2 [52]. The phosphorylation of ERK through $\beta$-arrestin 2 mediates the src activation, which then potentiates BDNF-stimulated TrkB signaling possibly by trafficking TrkB receptors to neuronal membranes [34, 53]. XYS may increase the phosphorylation of ERK to activate the TrkB pathway, thus alleviating the depressive behavior [54].

In conclusion, social isolation and CUMS induce depressive behavior by upregulating CORT and UCN2. Accordingly, the vicious cycle of HPA hyperactivity deteriorates and causes hippocampal neuron cell body injury. XYS improves the depressive-like behavior through the $\beta$-arrestin 2 and PP2Amediated downregulation of CRHR2 and the upregulation of BDNF and mTOR [8]. Further studies will be conducted 


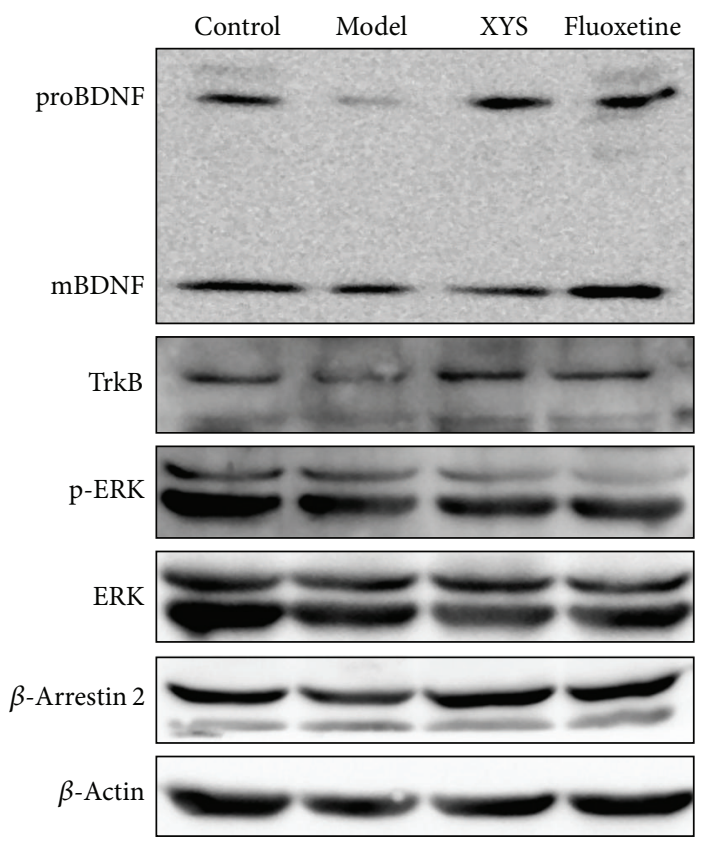

(a)
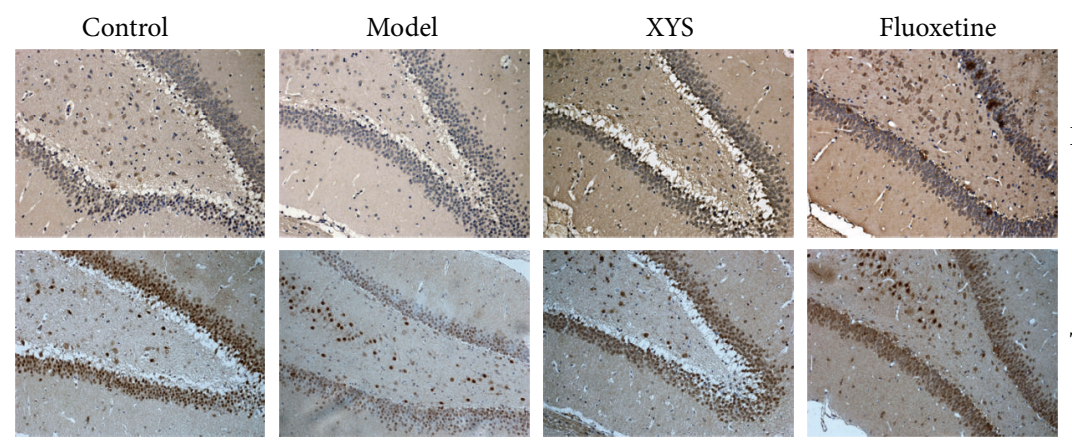

BDNF
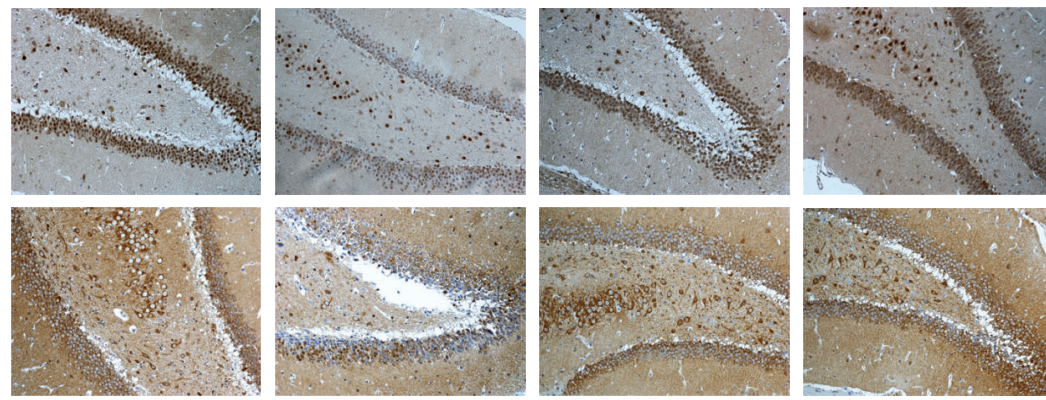

$\operatorname{TrkB}$
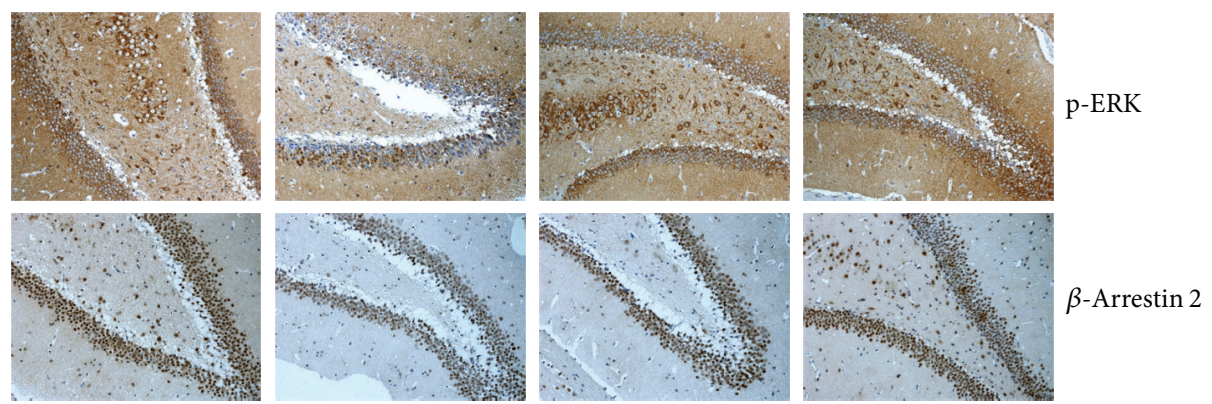

(b)

Figure 7: Representative Western blot analysis (a) and immunohistochemical staining (b) of BDNF, TrkB, p-ERK, ERK, and $\beta$-arrestin 2 in the hippocampus. BDNF: brain-derived neurotrophic factor; TrkB: tyrosine kinase receptor B; ERK: extracellular signal-regulated kinase. Refer to Table 2 for the semiquantitative analysis of the above images.

in vitro to investigate the mechanism of XYS in $\beta$-arrestin 2 -mediated complex formation $[42,44]$ in regulating GPCR desensitization and scaffolding.

\section{Conflict of Interests}

The authors declare that there is no conflict of interests regarding the publication of this paper.

\section{Authors' Contribution}

Xiaoxia Zhu, Oudong Xia, and Weili Han contributed equally to this paper.

\section{Acknowledgments}

This Project was supported by Grants from National Natural Sciences Foundation of China (nos. 81173168 and 81230085); 
TABLE 1: MALDI-TOF MS identification of protein molecules with altered expression.

\begin{tabular}{|c|c|c|c|c|c|c|}
\hline Number $^{\mathrm{a}}$ & Accession number ${ }^{\mathrm{b}}$ & $\begin{array}{l}\text { Molecular } \\
\text { weight }^{c}\end{array}$ & $p \mathrm{I}^{\mathrm{c}}$ & Score $^{\mathrm{d}}$ & Protein name & $\begin{array}{c}\text { Expression } \\
\text { level }^{\mathrm{e}}\end{array}$ \\
\hline 1 & P67779/PHB_RAT & 29859 & 5.57 & $57(51)$ & Prohibitin & $\uparrow, \Uparrow$ \\
\hline 2 & P28073/PSB6_RAT & 25502 & 4.85 & $39(26)$ & $\begin{array}{l}\text { Proteasome subunit beta } \\
\text { type- } 6\end{array}$ & $\uparrow, \Uparrow$ \\
\hline 3 & P68370/TBA1A_RAT & 50788 & 4.94 & $64(25)$ & Tubulin alpha-1A chain & $\downarrow, \Downarrow$ \\
\hline 4 & $N / A^{f}$ & & & & & $\downarrow, \Downarrow$ \\
\hline 5 & $\mathrm{~N} / \mathrm{A}$ & & & & & $\downarrow, \Downarrow$ \\
\hline 6 & Q4V8B2/DCNL3_RAT & 34899 & 5.07 & $60(51)$ & DCN1-like protein 3 & $\downarrow, \Downarrow$ \\
\hline 7 & P36876/2ABA_RAT & 52159 & 5.82 & $55(51)$ & $\begin{array}{l}\text { Serine/threonine-protein } \\
\text { phosphatase } 2 \mathrm{~A} 55 \mathrm{kDa} \\
\text { regulatory subunit B alpha } \\
\text { isoform }\end{array}$ & $\downarrow, \Downarrow$ \\
\hline 8 & Q6P9T8/TBB2C_RAT & 50225 & 4.79 & $83(26)$ & Tubulin beta-2C chain & $\downarrow, \Downarrow$ \\
\hline 9 & $\mathrm{~N} / \mathrm{A}$ & & & & & $\downarrow, \Downarrow$ \\
\hline 10 & Q8CJ99/SCLT1_RAT & 80622 & 5.98 & $52(51)$ & $\begin{array}{l}\text { Sodium channel and } \\
\text { clathrin linker } 1\end{array}$ & $\downarrow, \Downarrow$ \\
\hline
\end{tabular}

MALDI-TOF MS: matrix-assisted laser desorption/ionization time of flight mass spectrometer.

a defined according to spot positions in 2DE gel indicated, as in Figure 4.

b defined from http://www.uniprot.org/.

${ }^{c}$ Molecular weight value calculated by amino acid count; $p$ I value calculated from the database entry without any processing.

${ }^{\mathrm{d}}$ Protein scores are derived from ions scores as a nonprobabilistic basis for ranking protein hits. Protein scores greater than threshold value are significant $(P<$ 0.05).

e $\uparrow \downarrow$ Expression of sham group compared to that of model group; $\Uparrow \Downarrow$ expression of XYS group compared to that of model group.

${ }^{\mathrm{f}}$ No effective peaks of peptide mass fingerprinting were available.

TABLE 2: Semiquantitative analysis of protein expression and phosphorylation levels by Western blot (WB) and immunohistochemistry (IHC) $($ mean $\pm \mathrm{SD})$.

\begin{tabular}{|c|c|c|c|c|}
\hline & Control & Model & XYS & Fluoxetine \\
\hline BDNF-WB & $1.65 \pm 0.14$ & $1.32 \pm 0.06^{\mathbf{4}}$ & $1.49 \pm 0.06^{*}$ & $1.56 \pm 0.08^{*}$ \\
\hline TrkB-WB & $1.36 \pm 0.08$ & $0.87 \pm 0.06^{\mathbf{\Lambda}}$ & $1.53 \pm 0.06^{\triangle}$ & $1.37 \pm 0.10^{\#}$ \\
\hline p-ERK-WB & $1.29 \pm 0.05$ & $0.90 \pm 0.11^{\wedge}$ & $1.06 \pm 0.08^{\mathbf{\Delta}}$ & $1.18 \pm 0.08^{\#}$ \\
\hline ERK-WB & $1.15 \pm 0.07$ & $1.10 \pm 0.05$ & $1.09 \pm 0.05$ & $1.13 \pm 0.07$ \\
\hline$\beta$-Arrestin 2 & $2.05 \pm 0.11$ & $1.41 \pm 0.09^{\mathbf{}}$ & $1.75 \pm 0.04^{\mathbf{\Delta} \#}$ & $1.80 \pm 0.04^{\mathbf{\#}}$ \\
\hline p-mTOR-WB & $0.81 \pm 0.04$ & $0.28 \pm 0.04^{\mathbf{\Delta}}$ & $0.76 \pm 0.03^{\#}$ & $0.84 \pm 0.03^{\#}$ \\
\hline mTOR-WB & $1.22 \pm 0.06$ & $1.27 \pm 0.05$ & $1.22 \pm 0.05$ & $1.23 \pm 0.04$ \\
\hline p-Akt-WB & $0.99 \pm 0.05$ & $0.73 \pm 0.05^{\mathbf{\Lambda}}$ & $1.06 \pm 0.04^{\#}$ & $0.95 \pm 0.06^{\#}$ \\
\hline Akt-WB & $1.00 \pm 0.08$ & $1.13 \pm 0.08$ & $1.04 \pm 0.07$ & $1.09 \pm 0.08$ \\
\hline PP2A b-WB & $1.23 \pm 0.06$ & $1.37 \pm 0.02^{\triangle}$ & $1.13 \pm 0.04^{\#}$ & $1.19 \pm 0.10^{\#}$ \\
\hline PP2A c-WB & $3.06 \pm 0.09$ & $3.16 \pm 0.10$ & $3.03 \pm 0.07$ & $3.18 \pm 0.05$ \\
\hline CRHR1-WB & $1.54 \pm 0.06$ & $1.67 \pm 0.14$ & $1.61 \pm 0.09$ & $1.70 \pm 0.03$ \\
\hline CRHR2-WB & $0.36 \pm 0.02$ & $1.85 \pm 0.04^{\mathbf{\Lambda}}$ & $1.34 \pm 0.02^{\#}$ & $0.42 \pm 0.03^{\triangle \#}$ \\
\hline BDNF-IHC & $4.07 \pm 0.83$ & $1.37 \pm 0.52^{\mathbf{\Delta}}$ & $4.48 \pm 0.89^{\#}$ & $4.97 \pm 1.02^{\#}$ \\
\hline TrkB-IHC & $7.52 \pm 1.06$ & $1.60 \pm 0.54^{\mathbf{\Delta}}$ & $5.38 \pm 1.13^{\triangle \#}$ & $5.95 \pm 1.35^{\#}$ \\
\hline p-ERK-IHC & $7.07 \pm 1.36$ & $2.42 \pm 0.79^{\mathbf{\Lambda}}$ & $5.80 \pm 1.25^{\#}$ & $5.80 \pm 1.04^{\#}$ \\
\hline$\beta$-Arrestin 2-IHC & $8.27 \pm 1.01$ & $2.77 \pm 0.64^{\mathbf{A}}$ & $6.58 \pm 1.33^{\triangle \#}$ & $6.72 \pm 1.17^{\triangle \#}$ \\
\hline CRHR1-IHC & $5.67 \pm 1.23$ & $6.25 \pm 1.02$ & $5.47 \pm 1.26$ & $6.18 \pm 1.17$ \\
\hline CRHR2-IHC & $2.87 \pm 0.93$ & $4.27 \pm 0.74^{\boldsymbol{\Lambda}}$ & $2.58 \pm 0.79^{\#}$ & $2.32 \pm 0.63^{\#}$ \\
\hline
\end{tabular}

${ }^{\triangle} P<0.05,{ }^{\wedge} P<0.01$ versus control, ${ }^{*} P<0.05,{ }^{\#} P<0.01$ versus model. 
Science and Technology Project of Guangzhou (no. 12C32121551); Construction Traditional Chinese Medicine Province Research Projects of TCM Bureau of Guangdong Province (20121121).

\section{References}

[1] D. S. Hasin, R. D. Goodwin, F. S. Stinson, and B. F. Grant, "Epidemiology of major depressive disorder: results from the National Epidemiologic Survey on Alcoholism and Related Conditions," Archives of General Psychiatry, vol. 62, no. 10, pp. 1097-1106, 2005.

[2] U. B. O. D. Collaborators, "The state of US health, 1990-2010: burden of diseases, injuries, and risk factors," The Journal of the American Medical Association, vol. 310, no. 6, pp. 591-608, 2013.

[3] B. H. Hidaka, "Depression as a disease of modernity: explanations for increasing prevalence," Journal of Affective Disorders, vol. 140, no. 3, pp. 205-214, 2012.

[4] A. Gardner and R. G. Boles, "Beyond the serotonin hypothesis: Mitochondria, inflammation and neurodegeneration in major depression and affective spectrum disorders," Progress in NeuroPsychopharmacology and Biological Psychiatry, vol. 35, no. 3, pp. 730-743, 2011.

[5] Y. Chen, C. M. L. Kelton, Y. Jing, J. J. Guo, X. Li, and N. C. Patel, "Utilization, price, and spending trends for antidepressants in the US Medicaid program," Research in Social and Administrative Pharmacy, vol. 4, no. 3, pp. 244-257, 2008.

[6] P. L. Delgado, "Monoamine depletion studies: Implications for antidepressant discontinuation syndrome," Journal of Clinical Psychiatry, vol. 67, supplement 4, pp. 22-26, 2006.

[7] C. Schüle, T. C. Baghai, D. Eser, and R. Rupprecht, "Hypothalamic-pituitary-adrenocortical system dysregulation and new treatment strategies in depression," Expert Review of $\mathrm{Neu}$ rotherapeutics, vol. 9, no. 7, pp. 1005-1019, 2009.

[8] F. Pilar-Cuellar, R. Vidal, A. Diaz et al., "Neural plasticity and proliferation in the generation of antidepressant effects: hippocampal implication," Neural Plasticity, vol. 2013, Article ID 537265, 21 pages, 2013.

[9] R. S. Duman, J. Malberg, S. Nakagawa, and C. D’Sa, "Neuronal plasticity and survival in mood disorders," Biological Psychiatry, vol. 48, no. 8, pp. 732-739, 2000.

[10] R. Massart, R. Mongeau, and L. Lanfumey, "Beyond the monoaminergic hypothesis: neuroplasticity and epigenetic changes in a transgenic mouse model of depression," Philosophical Transactions of the Royal Society B: Biological Sciences, vol. 367, no. 1601, pp. 2485-2494, 2012.

[11] Z. Huang, Q. Mao, X. Zhong, Z. Li, F. Qiu, and S. Ip, "Mechanistic study on the antidepressant-like effect of Danggui-ShaoyaoSan, a chinese herbal formula," Evidence-Based Complementary and Alternative Medicine, vol. 2012, Article ID 173565, 7 pages, 2012.

[12] K. Y. Zhu, Q. Mao, S. Ip et al., "A standardized chinese herbal decoction, kai-xin-san, restores decreased levels of neurotransmitters and neurotrophic factors in the brain of chronic stressinduced depressive rats," Evidence-Based Complementary and Alternative Medicine, vol. 2012, Article ID 149256, 13 pages, 2012.

[13] Y. Chen, J. Liu, X. Wu, and E. C. Nice, "Xiangshao granule exerts antidepressive effects in a depression mouse model by ameliorating deficits in hippocampal BDNF and TrkB," Evidence-Based Complementary and Alternative Medicine, vol. 2013, Article ID 309262, 6 pages, 2013.
[14] Y. Zhang, M. Han, Z. Liu, J. Wang, Q. He, and J. Liu, "Chinese herbal formula Xiao Yao San for treatment of depression: a systematic review of randomized controlled trials," EvidenceBased Complementary and Alternative Medicine, vol. 2012, Article ID 931636, 13 pages, 2012.

[15] L. Bao, J. Chen, L. Huang et al., "Effects of Xiaoyao Wan on the behavioral despair and stress depression mice," Zhong Yao Cai, vol. 31, no. 9, pp. 1360-1364, 2008.

[16] J. X. Chen and Y. T. Tang, "Effect of Xiaoyao powder on changes of relative brain zone CRF gene expression in chronic restrained stress rats," Zhongguo Ying Yong Sheng Li Xue Za Zhi, vol. 20, no. 1, pp. 71-74, 2004.

[17] Q. L. Zhang, G. X. Yue, and Z. F. Wang, "Changes of BDNF in different encephalic region of rats of chronic immobilization stress model and effect of xiaoyaosan," Liaoning Journal of Traditional Chinese Medicine, vol. 37, no. 1, pp. 162-165, 2010.

[18] Y. C. Zhou, B. Liu, Y. J. Li et al., "Effects of buyang huanwu decoction on ventricular remodeling and differential protein profile in a rat model of myocardial infarction," Evidence-Based Complementary and Alternative Medicine, vol. 2012, Article ID 385247, 11 pages, 2012.

[19] Q. Q. Mao, S. P. Ip, K. M. Ko, S. H. Tsai, and C. T. Che, "Peony glycosides produce antidepressant-like action in mice exposed to chronic unpredictable mild stress: Effects on hypothalamicpituitary-adrenal function and brain-derived neurotrophic factor," Progress in Neuro-Psychopharmacology and Biological Psychiatry, vol. 33, no. 7, pp. 1211-1216, 2009.

[20] J. L. Pawluski, S. E. Lieblich, and L. A. Galea, "Offspring-exposure reduces depressive-like behaviour in the parturient female rat," Behavioural Brain Research, vol. 197, no. 1, pp. 55-61, 2009.

[21] T. Strekalova, R. Spanagel, D. Bartsch, F. A. Henn, and P. Gass, "Stress-induced anhedonia in mice is associated with deficits in forced swimming and exploration," Neuropsychopharmacology, vol. 29, no. 11, pp. 2007-2017, 2004.

[22] G. Lucca, C. M. Comim, S. S. Valvassori et al., "Increased oxidative stress in submitochondrial particles into the brain of rats submitted to the chronic mild stress paradigm," Journal of Psychiatric Research, vol. 43, no. 9, pp. 864-869, 2009.

[23] X. Sun, X. Zhong, Z. Liu et al., "Proteomic analysis of chronic restraint stress-induced Gan (fj)-stagnancy syndrome in rats," Chinese Journal of Integrative Medicine, vol. 16, no. 6, pp. 510$517,2010$.

[24] H. B. Cai, X. G. Sun, Z. F. Liu et al., "Effects of dahuangzhechong pills on cytokines and mitogen activated protein kinase activation in rats with hepatic fibrosis," Journal of Ethnopharmacology, vol. 132, no. 1, pp. 157-164, 2010.

[25] Y. J. Yang, Y. J. Su, D. T. Wang et al., "Tanshinol attenuates the deleterious effects of oxidative stress on osteoblastic differentiation via Wnt/FoxO3a signaling," Oxidative Medicine and Cellular Longevity, vol. 2013, Article ID 351895, 18 pages, 2013.

[26] R. J. Katz, "Animal model of depression: pharmacological sensitivity of a hedonic deficit," Pharmacology Biochemistry and Behavior, vol. 16, no. 6, pp. 965-968, 1982.

[27] P. Willner, "The validity of animal models of depression," Psychopharmacology, vol. 83, no. 1, pp. 1-16, 1984.

[28] X. Ma, D. Jiang, W. Jiang et al., "Social isolation-induced aggression potentiates anxiety and depressive-like behavior in male mice subjected to unpredictable chronic mild stress," PLoS ONE, vol. 6, no. 6, Article ID e20955, 2011.

[29] R. M. Jin, L. Huang, W. Zhou et al., "Study of Xiaoyao tablet on improving intestinal movement and other related function in 
normal mice or mice with insufficiency of the spleen," Chinese Journal of China Rehabilitation, vol. 7, no. 24, pp. 3316-3317, 2003.

[30] G. MacQueen and T. Frodl, "The hippocampus in major depression: Evidence for the convergence of the bench and bedside in psychiatric research," Molecular Psychiatry, vol. 16, no. 3, pp. 252-264, 2011.

[31] S. C. Caetano, J. P. Hatch, P. Brambilla et al., "Anatomical MRI study of hippocampus and amygdala in patients with current and remitted major depression," Psychiatry Research: Neuroimaging, vol. 132, no. 2, pp. 141-147, 2004.

[32] S. Campbell and G. MacQueen, "The role of the hippocampus in the pathophysiology of major depression," Journal of Psychiatry and Neuroscience, vol. 29, no. 6, pp. 417-426, 2004.

[33] D. H. Oh, Y. C. Park, and S. H. Kim, "Increased glycogen synthase kinase- $3 \beta$ mRNA level in the hippocampus of patients with major depression: A study using the Stanley neuropathology consortium integrative database," Psychiatry Investigation, vol. 7, no. 3, pp. 202-207, 2010.

[34] R. L. Hauger, V. Risbrough, R. H. Oakley, J. A. Olivares-Reyes, and F. M. Dautzenberg, "Role of CRF receptor signaling in stress vulnerability, anxiety, and depression," Annals of the New York Academy of Sciences, vol. 1179, pp. 120-143, 2009.

[35] Z. Bagosi, K. Csabafi, M. Palotai et al., "The interaction of Urocortin II and Urocortin III with amygdalar and hypothalamic cotricotropin-releasing factor (CRF) - reflections on the regulation of the hypothalamic-pituitary-adrenal (HPA) axis," Neuropeptides, vol. 47, no. 5, pp. 333-338, 2013.

[36] A. Skórzewska, A. Bidziński, M. Lehner et al., “The localization of brain sites of anxiogenic-like effects of urocortin-2," Neuropeptides, vol. 45, no. 1, pp. 83-92, 2011.

[37] S. C. Coste, S. E. Murray, and M. P. Stenzel-Poore, "Animal models of $\mathrm{CRH}$ excess and $\mathrm{CRH}$ receptor deficiency display altered adaptations to stress," Peptides, vol. 22, no. 5, pp. 733$741,2001$.

[38] V. Janssens and J. Goris, "Protein phosphatase 2A: a highly regulated family of serine/threonine phosphatases implicated in cell growth and signalling," Biochemical Journal, vol. 353, no. 3, pp. 417-439, 2001.

[39] J. Chen and X. Huang, "The PI3K/Akt pathway may play a key role in social isolation-caused schizophrenia comment re: Increased dopamine D2High receptors in rats reared in social isolation," Synapse, vol. 64, no. 6, pp. 486-487, 2010.

[40] L. Li, C. H. Ren, S. A. Tahir, C. Ren, and T. C. Thompson, "Caveolin-1 maintains activated Akt in prostate cancer cells through scaffolding domain binding site interactions with and inhibition of serine/ threonine protein phosphatases PP1 and PP2A," Molecular and Cellular Biology, vol. 23, no. 24, pp. 93899404, 2003.

[41] J. M. Horwood, F. Dufour, S. Laroche, and S. Davis, "Signalling mechanisms mediated by the phosphoinositide 3-kinase/Akt cascade in synaptic plasticity and memory in the rat," European Journal of Neuroscience, vol. 23, no. 12, pp. 3375-3384, 2006.

[42] J. M. Beaulieu, T. D. Sotnikova, S. Marion, R. J. Lefkowitz, R. R. Gainetdinov, and M. G. Caron, "An Akt/ $\beta$-arrestin 2/PP2A signaling complex mediates dopaminergic neurotransmission and behavior," Cell, vol. 122, no. 2, pp. 261-273, 2005.

[43] J. M. Beaulieu, S. Marion, R. M. Rodriguiz et al., "A $\beta$-arrestin 2 signaling complex mediates lithium action on behavior," Cell, vol. 132, no. 1, pp. 125-136, 2008.
[44] B. Luan, J. Zhao, H. Wu et al., "Deficiency of a B-arrestin-2 signal complex contributes to insulin resistance," Nature, vol. 457, no. 7233, pp. 1146-1149, 2009.

[45] T. Teli, D. Markovic, M. A. Levine, E. W. Hillhouse, and D. K. Grammatopoulos, "Regulation of corticotropin-releasing hormone receptor type $1 \alpha$ signaling: structural determinants for $G$ protein-coupled receptor kinase-mediated phosphorylation and agonist-mediated desensitization," Molecular Endocrinology, vol. 19, no. 2, pp. 474-490, 2005.

[46] R. L. Hauger, J. A. Olivares-Reyes, S. Braun et al., "Desensitization of human $\mathrm{CRF}_{2(a)}$ receptor signaling governed by agonist potency and $\beta$ arrestin2 recruitment," Regulatory Peptides, vol. 186, pp. 62-76, 2013.

[47] G. Schreiber, M. Golan, and S. Avissar, " $\beta$-arrestin signaling complex as a target for antidepressants and as a depression marker," Drug News and Perspectives, vol. 22, no. 8, pp. 467-480, 2009.

[48] R. L. Hauger, V. Risbrough, O. Brauns, and F. M. Dautzenberg, "Corticotropin releasing factor (CFR) receptor signaling in the central nervous system: new molecular targets," CNS and Neurological Disorders-Drug Targets, vol. 5, no. 4, pp. 453-479, 2006.

[49] G. R. Valdez, "CRF receptos as a potential target in the development of novel pharmacotherapies for depression," Current Pharmaceutical Design, vol. 15, no. 14, pp. 1587-1594, 2009.

[50] S. Scaccianoce, P. Del Bianco, G. Paolone et al., "Social isolation selectively reduces hippocampal brain-derived neurotrophic factor without altering plasma corticosterone," Behavioural Brain Research, vol. 168, no. 2, pp. 323-325, 2006.

[51] X. Qi, W. Lin, D. Wang, Y. Pan, W. Wang, and M. Sun, "A role for the extracellular signal-regulated kinase signal pathway in depressive-like behavior," Behavioural Brain Research, vol. 199, no. 2, pp. 203-209, 2009.

[52] X. Qi, W. Lin, J. Li et al., "Fluoxetine increases the activity of the ERK-CREB signal system and alleviates the depressive-like behavior in rats exposed to chronic forced swim stress," Neurobiology of Disease, vol. 31, no. 2, pp. 278-285, 2008.

[53] S. Traver, M. Marien, E. Martin, E. C. Hirsch, and P. P. Michel, "The phenotypic differentiation of locus ceruleus noradrenergic neurons mediated by brain-derived neurotrophic factor is enhanced by corticotropin releasing factor through the activation of a cAMP-dependent signaling pathway," Molecular Pharmacology, vol. 70, no. 1, pp. 30-40, 2006.

[54] C. Chen, L. Hsu, L. Huang, and T. Huang, "Chronic administration of cyclosporine a changes expression of BDNF and TrkB in rat hippocampus and midbrain," Neurochemical Research, vol. 35, no. 7, pp. 1098-1104, 2010. 


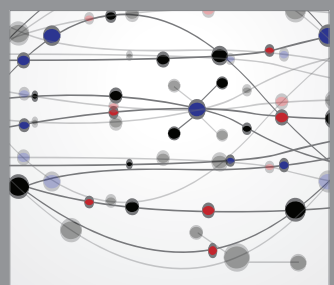

The Scientific World Journal
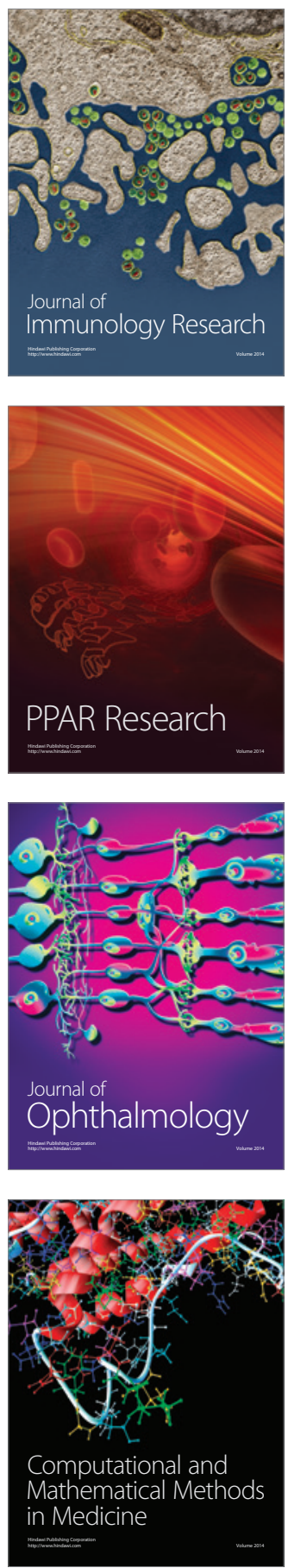

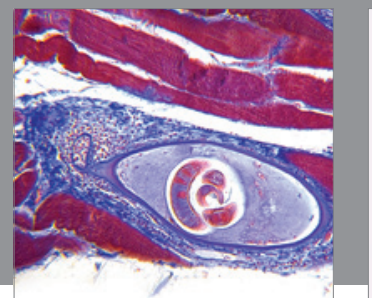

Gastroenterology

Research and Practice
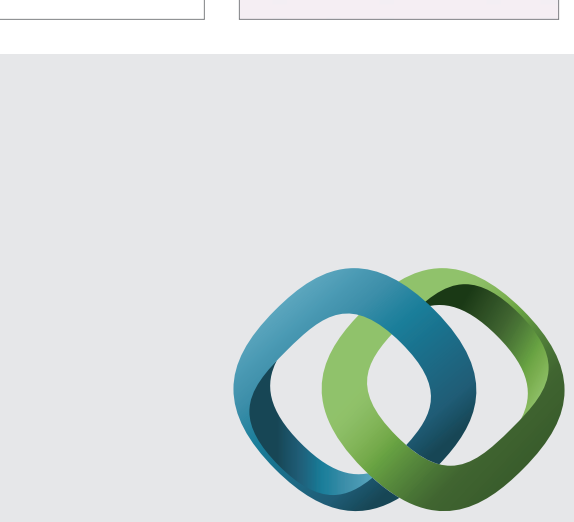

\section{Hindawi}

Submit your manuscripts at

http://www.hindawi.com
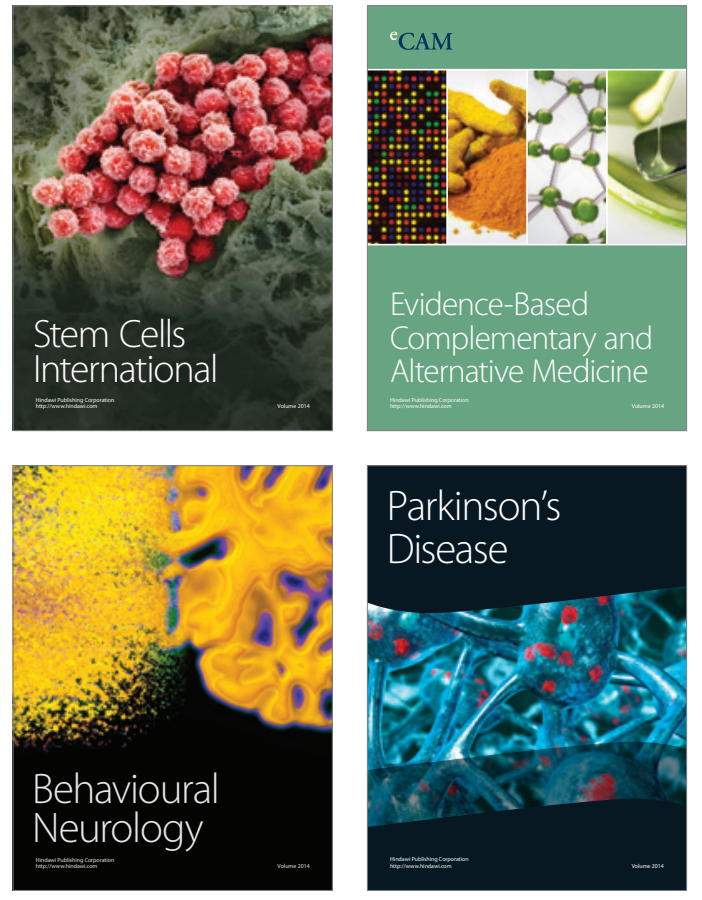
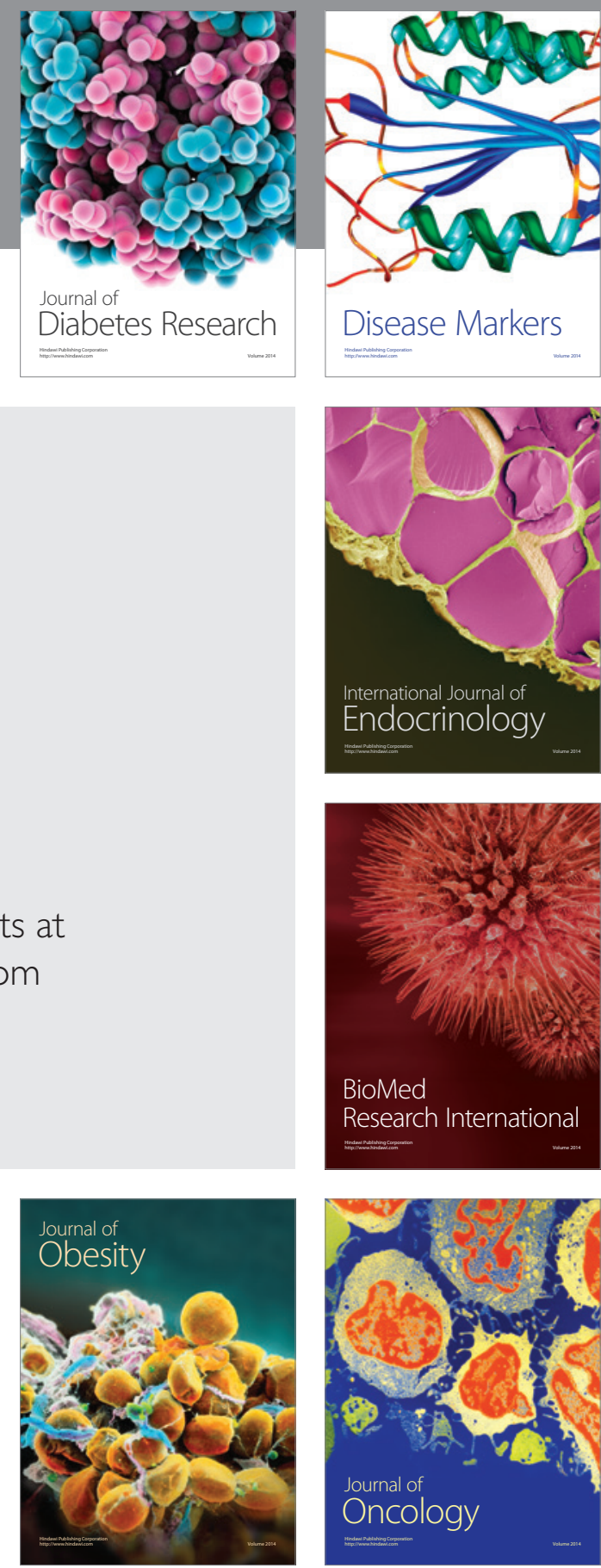

Disease Markers
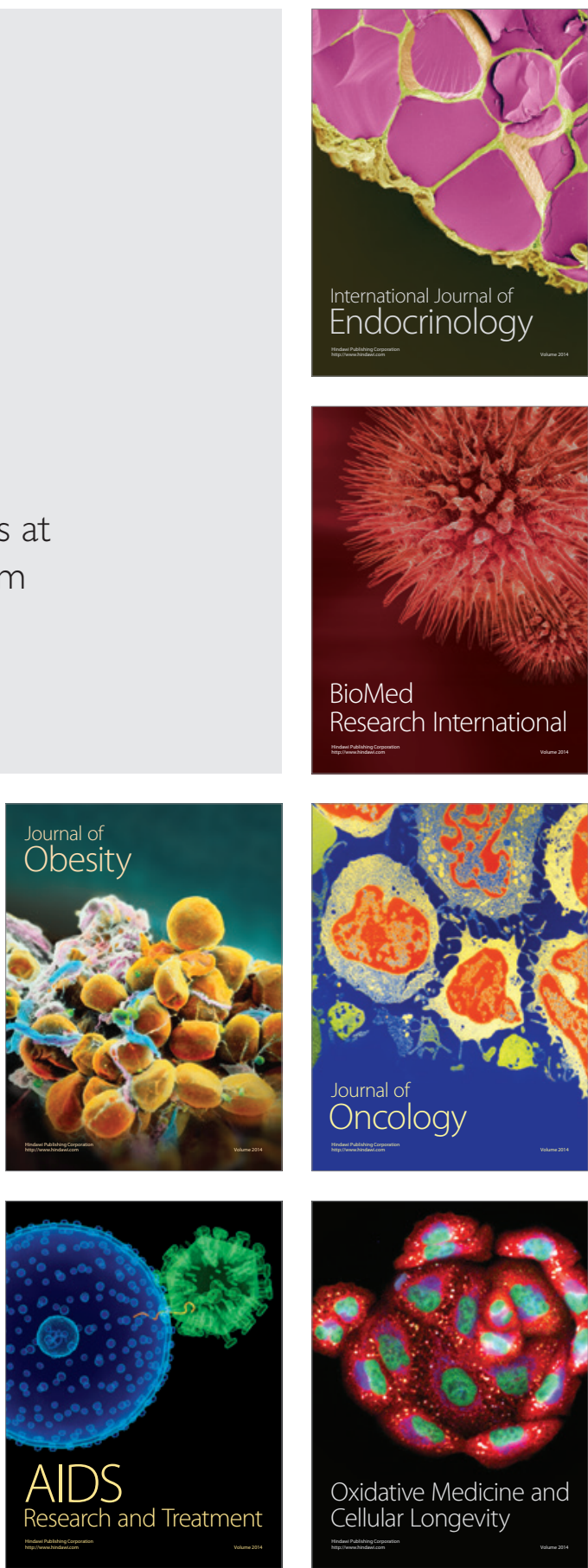